Pacific

Journal of

Mathematics

MINIMAL SURFACES OF RIEMANN TYPE IN THREE-DIMENSIONAL PRODUCT MANIFOLDS

LAURENT HAUSWIRTH 


\title{
MINIMAL SURFACES OF RIEMANN TYPE IN THREE-DIMENSIONAL PRODUCT MANIFOLDS
}

\author{
LAURENT HAUSWIRTH
}

\begin{abstract}
We construct and classify minimal surfaces foliated by horizontal curves of constant curvature in $\mathbb{H}^{2} \times \mathbb{R}, \mathbb{R}^{2} \times \mathbb{R}$ and $\mathbb{S}^{2} \times \mathbb{R}$. The main tool is the existence of a Shiffman Jacobi field; such fields characterize the property of being foliated by circles in these product manifolds.
\end{abstract}

\section{Introduction}

We are interested in minimal surfaces properly embedded in the product space $M \times \mathbb{R}$, where $M$ is a complete Riemannian surface with constant curvature $c_{0}$. The main examples are $M=\mathbb{H}^{2}, \mathbb{R}^{2}, \mathbb{S}^{2}$. When $c_{0}=0$, this is the theory of periodic (singly, doubly, and triply) minimal surfaces in $\mathbb{R}^{3}$ and has been well developed; see [Meeks 1990; Meeks et al. 1998; 2004; Meeks and Rosenberg 1989]. For general $M$, the theory was initiated by Rosenberg [2002] and developed in [Nelli and Rosenberg 2002; Meeks and Rosenberg 2004, 2005]. These authors have found a rich family of examples including helicoids, catenoids and unduloids (surfaces of genus zero). By solving Plateau problems, they construct examples of higher topological type inspired by the classical theory in $\mathbb{R}^{3}$.

Examples are so numerous that we intend to classify some of them. This paper is devoted to annuli minimal surfaces properly embedded in product spaces and transverse to $M \times\{t\}$ for every $t \in \mathbb{R}$. We classify and construct all examples foliated by constant curvature horizontal curves, a two parameter family in each $M \times \mathbb{R}$. With the exception of catenoids in $\mathbb{H}^{2} \times \mathbb{R}$, they are all simply periodic, that is, properly embedded in the quotient space $(M \times \mathbb{R}) / T$, where $T$ is a vertical translation or screw motion.

The main point is to present a unified point of view. Our computations and parametrizations are transversal to each product space and contain the classical theory of $\mathbb{R}^{3}$.

MSC2000: 49Q05, 53C42, 53A10, 57R40, 53C43, 58E20.

Keywords: periodic minimal surface, harmonic map, stable minimal surface, stability index, Jacobi field, Jacobi operator. 
Riemann constructed and classified examples of minimal surfaces foliated by straight lines and circles in horizontal planes of $\mathbb{R}^{3}$. He construct a family of minimal annuli with an infinite number of parallel flat ends distributed in a periodic way along the vertical. We generalize this construction to the case where the ambient space is $\mathbb{H}^{2} \times \mathbb{R}$ and $\mathbb{S}^{2} \times \mathbb{R}$ (Section 3 ).

In Section 2, we generalize the beautiful work of M. Shiffman [1956], finding a Jacobi field derived from the derivative of the curvature of the horizontal curves. Such a Jacobi field was used and explained by Y. Fang [1994] to characterize Riemann's examples in $\mathbb{R}^{3}$ as the unique properly embedded compact annuli bounded by two circles. Fang and Wei [1998] extend this uniqueness result to the case where the minimal annulus is bounded by two straight lines or circle with finite total curvature less than $12 \pi$.

Shiffman's Jacobi field is an important ingredient in the study of the uniqueness conjecture of the Riemann example. In Theorem 2.4, in the spirit of [Fang 1994], we prove an uniqueness result for compact annuli $A$ having a Jacobi operator $L$ of index at most 1:

Main Theorem. Let $A$ be a compact minimal annulus embedded in $M \times \mathbb{R}$, where $M$ is $\mathbb{M}^{2}, \mathbb{R}^{2}$ or $\mathbb{S}^{2}$, bounded by two curves of constant curvature in $M \times\left\{t_{1}\right\}$ and $M \times\left\{t_{2}\right\}$. If the corresponding Jacobi operator L has index at most 1 , then $A$ is foliated by circles or geodesics; that is, $A \cap(M \times\{t\})$ is a curve with constant curvature $k_{g}$ for all $t \in\left[t_{1}, t_{2}\right]$.

In Section 3 we are inspired by the work of Abresch [1987] on constant mean curvature tori in $\mathbb{R}^{3}$ to represent our examples by periodic elliptic functions. Then we construct and classify a two-parameter family of minimal surfaces foliated by constant curvature curves in horizontal sections. In particular we find a parametrization of Riemann's classical example, which has been used to understand the Jacobi operator in a forthcoming paper [Hauswirth and Pacard 2005]. R. Sá Earp and E. Toubiana [2004] have constructed other minimal examples invariant under screw motion and B. Daniel [2004] has explained the Gauss-Codazzi equations. In this last work he found interesting formulas and geometric properties of some examples described in this paper.

\section{Minimal annuli transverse to horizontal planes}

Let $A \subset \mathbb{C}$ be a domain and let $M$ be a complete Riemannian two-manifold with metric $g$ and (not necessarily constant) Gaussian curvature $K_{M}$. We consider an embedded minimal surface $X=(F, h): A \subset \mathbb{R}^{2} \rightarrow M \times \mathbb{R}$, transverse to $M \times\{t\}$ for every $t \in \mathbb{R}$. Assume that $M$ is isometrically embedded in $\mathbb{R}^{k}$, for $k$ large enough. Now $F: A \rightarrow M \subset \mathbb{R}^{k}$ has coordinates $F=\left(F_{1}, F_{2}, \ldots, F_{k}\right)$ in $\mathbb{R}^{k}$. By definition (see [Lawson 1980]) the mean curvature vector in $\mathbb{R}^{k}$ is 
$\vec{H}=(\triangle X)^{T_{X} M \times \mathbb{R}}=\left((\triangle F)^{T_{F} M}, \triangle x_{3}\right)=0$. Then $F: A \rightarrow M$ is a harmonic map between $A$ and the Riemannian surface $M$, while $h: A \rightarrow \mathbb{R}$ is a proper real harmonic function (see [Rosenberg 2002]).

The vertical coordinate $h$ (with its harmonic conjugate $h^{*}$ ) parametrizes the surface conformally. Indeed, $d h$ never vanishes, by the transversality assumption, so the holomorphic map $i\left(h+i h^{*}\right): A \rightarrow \mathbb{C}$ provides a conformal parameter $z=x+i y$ for $A$. In the following we will assume that $X(z)=(F(z), y)$ is a conformal embedding of $A$ with the metric $d s^{2}=\lambda(z)|d z|^{2}$ induced by $X$.

If $\left(U, \rho(u)|d u|^{2}\right)$ is a local parametrization of $M$, the harmonic map equation in the complex coordinate $u=u_{1}+i u_{2}$ of $M$ (see [Schoen and Yau 1997, p. 8]) is

$$
F_{z \bar{z}}+(\log \rho)_{u} F_{z} F_{\bar{z}}=0,
$$

where $2 F_{z}=F_{x}-i F_{y}$. Since $X=(F, y)$ is a conformal immersion, we have $\left|F_{x}\right|_{g}^{2}=\left|F_{y}\right|_{g}^{2}+1=\cosh ^{2} \omega$ and $\left\langle F_{x}, F_{y}\right\rangle_{g}=0$. Then the holomorphic quadratic Hopf differential is

$$
Q_{F}=\phi(z)(d z)^{2}=\frac{1}{4}\left(\left|F_{x}\right|_{g}^{2}-\left|F_{y}\right|_{g}^{2}+2 i\left\langle F_{x}, F_{y}\right\rangle_{g}\right)=\frac{1}{4}(d z)^{2} .
$$

Harmonic maps fulfill the Böchner formula, as is well known [Schoen and Yau 1997, p. 9]:

$$
\frac{1}{\lambda} \triangle_{0} \log \frac{\left|F_{z}\right|}{\left|F_{\bar{z}}\right|}=-2 K_{M} J(F)
$$

where $J(F)=\rho / \lambda\left(\left|F_{z}\right|^{2}-\left|F_{\bar{z}}\right|^{2}\right)$ is the Jacobian of $F$ with $\left|F_{z}\right|^{2}=F_{z} \bar{F}_{z}$.

We now prove that if we write the metric as $d s^{2}=\lambda|d z|^{2}=\cosh ^{2} \omega|d z|^{2}$, the Böchner formula becomes the sinh-Gordon equation for the function $\omega$ (see Proposition 2.1). This will give us a structure equation that we will use to study extrinsic properties of the surfaces.

Consider the projection $\Pi$ of $A$ on $M \times\{0\}$ and the level curves

$$
\begin{aligned}
& \gamma_{h}=A \cap(M \times\{y=h\})=F(x, h), \\
& \gamma_{v}=\Pi(\{x=v\})=F(v, y) .
\end{aligned}
$$

We derive general formulas for the geodesic curvature in $M$ of $\gamma_{h}$ and $\gamma_{v}$ (at points where $\gamma_{v}$ is not a singular curve) as a function of $\omega$ :

Proposition 2.1. Define the real function $\omega: A \rightarrow \mathbb{R}$ by $d s^{2}=\lambda|d z|^{2}=\cosh ^{2} \omega|d z|^{2}$. Then $\omega$ is a solution of the structure equation

$$
\triangle_{0} \omega+K_{M} \sinh \omega \cosh \omega=0
$$


where $\triangle_{0} \omega=\omega_{x x}+\omega_{y y}$. The geodesic curvature in $M$ of the level curves $\gamma_{h}$ and $\gamma_{v}$ is given by

$$
k_{g}\left(\gamma_{h}\right)=\frac{-\omega_{y}}{\cosh \omega} \quad \text { and }(\text { for } \omega \neq 0) \quad k_{g}\left(\gamma_{v}\right)=\frac{\omega_{x}}{\cosh \omega} \operatorname{coth} \omega .
$$

Proof. Since $X=(F, y)$ is a conformal immersion, we have $\left|F_{x}\right|_{g}^{2}=\left|F_{y}\right|_{g}^{2}+1=$ $\cosh ^{2} \omega$ and $\left\langle F_{x}, F_{y}\right\rangle_{g}=0$. Now let us consider $\left(U, \rho(u)|d u|^{2}\right)$ a local parametrization of $M$. We define the local function $\psi$ as the argument of $F_{x}$ :

$$
F_{x}=\frac{1}{\sqrt{\rho}} \cosh \omega e^{i \psi} \text { and } F_{y}=\frac{i}{\sqrt{\rho}} \sinh \omega e^{i \psi} .
$$

From the Böchner formula (2) we have

$$
\frac{1}{\lambda} \triangle_{0} \log \frac{\left|F_{z}\right|}{\left|F_{\bar{z}}\right|}=-2 K_{M} \frac{\rho}{\lambda}\left|F_{z}\right|\left|F_{\bar{z}}\right|\left(\frac{\left|F_{z}\right|}{\left|F_{\bar{z}}\right|}-\frac{\left|F_{\bar{z}}\right|}{\left|F_{z}\right|}\right) .
$$

But by a direct computation with $\rho\left|F_{x}\right|^{2}=\cosh ^{2} \omega$ and $\rho\left|F_{y}\right|^{2}=\sinh ^{2} \omega$ we derive

$$
\left|F_{z}\right|^{2}\left|F_{\bar{z}}\right|^{2}=\frac{1}{16}\left(\left(\left|F_{x}\right|^{2}-\left|F_{y}\right|^{2}\right)^{2}+4\left\langle F_{x}, F_{y}\right\rangle^{2}\right)=\frac{1}{16 \rho^{2}} .
$$

Now from (4) with $2 \sqrt{\rho}\left|F_{z}\right|=e^{\omega}$ and $2 \sqrt{\rho}\left|F_{\bar{z}}\right|=e^{-\omega}$, we derive the sinh-Gordon equation (3):

$$
\triangle_{0} \omega=-\frac{1}{2} K_{M} \sinh 2 \omega=-K_{M} \sinh \omega \cosh \omega .
$$

We consider the curves $\gamma_{h}$ and $\gamma_{v}$ parametrized in $\left(U, \rho(u)|d u|^{2}\right)$ with tangent vectors $F_{x}$ and $F_{y}$ respectively. If $k_{g}$ is the curvature of a curve $\gamma$ in $\left(U, \rho(u)|d u|^{2}\right)$ and $k_{e}$ is the Euclidean curvature in $\left(U,|d u|^{2}\right)$, we get, by a conformal change of the metric,

$$
k_{g}=\frac{k_{e}}{\sqrt{\rho}}-\frac{\langle\nabla \sqrt{\rho}, n\rangle}{\rho},
$$

where $n$ is the Euclidean normal to the curve $\gamma$. In particular $n=(-\sin \psi, \cos \psi)$ for the curve $\gamma_{h}$ ( $n$ is along $F_{y}$ ). If $s$ denotes the arclength of $\gamma_{h}$, we have

and

$$
k_{e}\left(\gamma_{h}\right)=\psi_{s}=\frac{\psi_{x} \sqrt{\rho}}{\cosh \omega}
$$

$$
\frac{\langle\nabla \sqrt{\rho}, n\rangle}{\rho}=\frac{\langle\nabla \log \sqrt{\rho}, n\rangle}{\sqrt{\rho}}=\frac{1}{2 \sqrt{\rho}}\left(\cos \psi(\log \rho)_{u_{2}}-\sin \psi(\log \rho)_{u_{1}}\right) .
$$

The tangent vector of $\gamma_{v}$ is $F_{y}$ which is zero at points where $\omega=0$. If $s$ denote the arclength of $\gamma_{v}$, we have

$$
k_{e}\left(\gamma_{v}\right)=\psi_{s}=\frac{\psi_{y} \sqrt{\rho}}{\sinh \omega}
$$


and with $n=(-\cos \psi,-\sin \psi)$,

$$
\frac{\langle\nabla \sqrt{\rho}, n\rangle}{\rho}=\frac{\langle\nabla \log \sqrt{\rho}, n\rangle}{\sqrt{\rho}}=-\frac{1}{2 \sqrt{\rho}}\left(\cos \psi(\log \rho)_{u_{1}}+\sin \psi(\log \rho)_{u_{2}}\right) .
$$

In summary we have

$$
\begin{aligned}
& k_{g}\left(\gamma_{h}\right)=\frac{\psi_{x}}{\cosh \omega}-\frac{1}{2 \sqrt{\rho}}\left(\cos \psi(\log \rho)_{u_{2}}-\sin \psi(\log \rho)_{u_{1}}\right), \\
& k_{g}\left(\gamma_{v}\right)=\frac{\psi_{y}}{\sinh \omega}+\frac{1}{2 \sqrt{\rho}}\left(\cos \psi(\log \rho)_{u_{1}}+\sin \psi(\log \rho)_{u_{2}}\right) .
\end{aligned}
$$

Now we compute $\psi_{x}$ as a function of $\omega_{y}$ and $\psi_{y}$ as a function of $\omega_{x}$. In the complex coordinate $z$ we have

$$
F_{z}=\frac{e^{\omega+i \psi}}{2 \sqrt{\rho}} \quad \text { and } \quad F_{\bar{z}}=\frac{e^{-\omega+i \psi}}{2 \sqrt{\rho}} .
$$

Placing these expressions in the harmonic equation (1) we derive

$$
(-\omega+i \psi)_{z}=-\sqrt{\rho}\left(\frac{1}{\sqrt{\rho}}\right)_{z}-(\log \rho)_{u} F_{z} .
$$

Now note that

$$
-\sqrt{\rho}\left(\frac{1}{\sqrt{\rho}}\right)_{z}=\frac{1}{2}(\log \rho)_{z}=\frac{1}{2}\left((\log \rho)_{u} F_{z}+(\log \rho)_{\bar{u}} \bar{F}_{z}\right)
$$
where $2(\log \rho)_{u}=(\log \rho)_{u_{1}}-i(\log \rho)_{u_{2}}$ and $\bar{F}_{z}=\frac{1}{2 \sqrt{\rho}} e^{-\omega-i \psi}$. Collecting these
equations we obtain

$$
(-\omega+i \psi)_{z}=\frac{1}{2}(\log \rho)_{\bar{u}} \bar{F}_{z}-\frac{1}{2}(\log \rho)_{u} F_{z} .
$$

The real and imaginary parts give

$$
\begin{aligned}
& \psi_{x}+\omega_{y}=\frac{\cosh \omega}{2 \sqrt{\rho}}\left(\cos \psi(\log \rho)_{u_{2}}-\sin \psi(\log \rho)_{u_{1}}\right), \\
& \psi_{y}-\omega_{x}=-\frac{\sinh \omega}{2 \sqrt{\rho}}\left(\cos \psi(\log \rho)_{u_{1}}+\sin \psi(\log \rho)_{u_{2}}\right) .
\end{aligned}
$$

Insert this into the expression for the curvature:

$$
\begin{aligned}
k_{g}\left(\gamma_{h}\right) & =\frac{\psi_{x}}{\cosh \omega}-\frac{1}{2 \sqrt{\rho}}\left(\cos \psi(\log \rho)_{u_{2}}-\sin \psi(\log \rho)_{u_{1}}\right)=\frac{-\omega_{y}}{\cosh \omega}, \\
k_{g}\left(\gamma_{v}\right) & =\frac{\psi_{y}}{\sinh \omega}+\frac{1}{2 \sqrt{\rho}}\left(\cos \psi(\log \rho)_{u_{1}}+\sin \psi(\log \rho)_{u_{2}}\right)=\frac{\omega_{x}}{\sinh \omega} \\
& =\frac{\omega_{x}}{\cosh \omega} \operatorname{coth} \omega .
\end{aligned}
$$


In the rest of this section we will consider only the geodesic curvature of $\gamma_{h}$ that we will denote by $k_{g}$. Now we generalize a result from [Shiffman 1956], to the effect that $\sqrt{\lambda}\left(k_{g}\right)_{x}$ is a Jacobi field. In particular, if $u$ is zero on $A$, the horizontal curves are of constant curvature.

Theorem 2.2. Let $A$ be a minimal surface embedded in a product space $M \times \mathbb{R}$ with $K_{M}=c_{0}$ a constant, and assume A transverse to every section $M \times\{t\}$. Then the function $u=-\cosh \omega\left(k_{g}\right)_{x}$ is a Jacobi field; that is, $u$ is solution of the elliptic equation:

$$
L u=\triangle_{g} u+\operatorname{Ric}(N) u+|d N|^{2} u=0,
$$

where $\operatorname{Ric}(N)$ is the Ricci curvature of the two planes tangent to $A,|d N|$ is the norm of the second fundamental form and $\triangle_{g}=(1 / \lambda) \triangle_{0}$.

Proof. Since $k_{g}\left(\gamma_{h}\right)=-\omega_{y} / \cosh \omega$ we have $u=\omega_{x y}-\tanh \omega \omega_{x} \omega_{y}$. We establish by a straightforward computation that

$$
\triangle_{0} u=u_{x x}+u_{y y}=-\left(c_{0}+2 \frac{|\nabla \omega|^{2}}{\cosh ^{2} \omega}\right) u
$$

which is $\lambda L u=0$. To prove (6) we compute $\operatorname{Ric}(N)$. Let $\left(e_{1}, e_{2}, e_{3}\right)$ be an oriented orthonormal frame in $M \times \mathbb{R}$. Then if $K\left(e_{i}, e_{j}\right)$ denotes the sectional curvature of the two-plane $\left(e_{i}, e_{j}\right)$ in $M \times \mathbb{R}$ and $S=K\left(e_{1}, e_{2}\right)+K\left(e_{1}, e_{3}\right)+K\left(e_{2}, e_{3}\right)=K_{M}=$ $c_{0}$ is the scalar curvature, we have the well-known formula

$$
\operatorname{Ric}(N)+|d N|^{2}=S+K\left(X_{x}, X_{y}\right)-2 K_{g} .
$$

Now compute the sectional curvature $K\left(X_{x}, X_{y}\right)$ of the tangent plane $T_{p} A$ :

$$
K\left(X_{x}, X_{y}\right)=\frac{\left\langle R\left(F_{x}, F_{y}+e_{3}\right) F_{x}, F_{y}+e_{3}\right\rangle}{\left|X_{x}\right|^{2}\left|X_{y}\right|^{2}-\left\langle X_{x}, X_{y}\right\rangle}=c_{0} \frac{\left|F_{x}\right|^{2}\left|F_{y}\right|^{2}}{\left|F_{x}\right|^{2}\left(\left|F_{y}\right|^{2}+1\right)}=c_{0}-\frac{c_{0}}{\lambda} .
$$

We plug $\lambda=\cosh ^{2} \omega$ into the expression of the Gauss curvature:

$$
K_{g}=-\frac{1}{2 \lambda} \triangle_{0} \log \lambda=-\frac{4}{\cosh ^{2} \omega}(\log \cosh \omega)_{z \bar{z}}=c_{0} \tanh ^{2} \omega-\left(\frac{\omega_{x}^{2}+\omega_{y}^{2}}{\cosh ^{4} \omega}\right) .
$$

Next we justify that (6) is $\lambda L u=0$ :

$\operatorname{Ric}(N)+|d N|^{2}=2 c_{0}-\frac{c_{0}}{\cosh ^{2} \omega}-2 c_{0} \tanh ^{2} \omega+2 \frac{|\nabla \omega|^{2}}{\cosh ^{4} \omega}=\frac{c_{0}}{\cosh ^{2} \omega}+2 \frac{|\nabla \omega|^{2}}{\cosh ^{4} \omega}$.

Now we prove (6) itself:

$\triangle_{0} u=\left(\triangle_{0} \omega\right)_{x y}-\left(\triangle_{0} \tanh \omega\right) \omega_{x} \omega_{y}-\tanh \omega\left(\triangle_{0} \omega_{x} \omega_{y}\right)$

$$
-2(\tanh \omega)_{x}\left(\omega_{x} \omega_{y}\right)_{x}-2(\tanh \omega)_{y}\left(\omega_{x} \omega_{y}\right)_{y} .
$$


Using the equality $\triangle_{0} \omega+c_{0} \sinh \omega \cosh \omega=0$, we have

$$
\begin{aligned}
\left(\triangle_{0} \omega\right)_{x y} & =\left(\frac{-c_{0}}{2} \sinh 2 \omega\right)_{x y}=-c_{0} \omega_{x y} \cosh 2 \omega-2 c_{0} \omega_{x} \omega_{y} \sinh 2 \omega, \\
\triangle_{0} \omega_{x} \omega_{y} & =-c_{0} \omega_{x y} \sinh 2 \omega-2 c_{0} \omega_{x} \omega_{y} \cosh 2 \omega, \\
\triangle_{0} \tanh \omega & =\left(-c_{0}-2 \frac{|\nabla \omega|^{2}}{\cosh ^{2} \omega}\right) \tanh \omega,
\end{aligned}
$$

and

$2(\tanh \omega)_{x}\left(\omega_{x} \omega_{y}\right)_{x}+2(\tanh \omega)_{y}\left(\omega_{x} \omega_{y}\right)_{y}=\frac{2 \omega_{x y}|\nabla \omega|^{2}-2 c_{0} \omega_{x} \omega_{y} \sinh \omega \cosh \omega}{\cosh ^{2} \omega}$.

Then

$$
\begin{aligned}
\triangle_{0} u=-c_{0}(\cosh 2 \omega-\tanh \omega \sinh 2 \omega) \omega_{x y} & \\
-2 \frac{|\nabla \omega|^{2}}{\cosh ^{2} \omega} \omega_{x y} & -2 c_{0} \omega_{x} \omega_{y}(\sinh 2 \omega-\tanh \omega \cosh 2 \omega) \\
& \quad-2 c_{0} \tanh \omega \omega_{x} \omega_{y}+\left(c_{0}+2 \frac{|\nabla \omega|^{2}}{\cosh ^{2} \omega}\right) \tanh \omega \omega_{x} \omega_{y} .
\end{aligned}
$$

Since $\cosh 2 \omega-\tanh \omega \sinh 2 \omega=1$ and $\tanh \omega \cosh 2 \omega-\sinh 2 \omega=-\tanh \omega$, we have proved (6).

Now with these Jacobi fields we derive a global result on annuli embedded in $M \times \mathbb{R}$. First we generalize a theorem of Shiffman [1956]:

Theorem 2.3. Let $A$ be a compact minimal annulus immersed in $M \times \mathbb{R}$ with $K_{M}=c_{0} \leq 0$. If $A$ is bounded by two curves $\Gamma_{1}$ and $\Gamma_{2}$ with positive geodesic curvature in $M \times\left\{t_{1}\right\}$ and $M \times\left\{t_{2}\right\}$, then $A$ is foliated by horizontal curves of positive curvature; that is, $A \cap(M \times\{t\})$ is a curve of curvature $k_{g}>0$.

Proof. This follows from the maximum principle and Proposition 2.1, applied to the linearized sinh-Gordon equation:

$$
\begin{aligned}
& \triangle_{0} \omega_{y}+K_{M} \omega_{y} \cosh 2 \omega=0 \quad \text { on } A \text {, } \\
& \omega_{y}<0 \quad \text { on } \partial A=\Gamma_{1} \cup \Gamma_{2} .
\end{aligned}
$$

Remark. For $K_{M}=c_{0}>0$, there are counterexamples: consider the compact part of an unduloid in $\mathbb{S}^{2} \times \mathbb{R}$ (page 103), which amounts to an annulus bounded by two circles of positive curvature and yet contains geodesics and negative curvature curves in its interior.

Now we generalize the geometric characterization of Shiffman [1956] and Fang [1994] for the annulus with low index bounded by constant curvature curves: 
Theorem 2.4. Let $A$ be a compact minimal annulus embedded in $M \times \mathbb{R}$ with $K_{M}=c_{0}$. We assume that the boundary $\partial A=\Gamma_{1} \cup \Gamma_{2}$ are curves with constant geodesic curvature in $M \times\left\{t_{1}\right\}$ and $M \times\left\{t_{2}\right\}$; that is, $u=0$ on $\partial A$.

If $M=\mathbb{H}^{2}, \mathbb{R}^{2}$ or $\mathbb{S}^{2}$ and $A$ satisfies $\operatorname{Index}(L) \leq 1$, then $u$ is identically zero and $A$ is foliated by horizontal curves of constant curvature in $M$.

If $M$ is not simply connected, the result is true with the additional hypothesis that $\operatorname{Index}(L)=0$ (A is stable).

Proof. (See [Fang 1994] for details.) By the four-vertex theorem, $u$ has four zeros on each horizontal Jordan curve of a simply connected space (see [Jackson 1945]) and then $u$ has at least four nodal domains on the annulus $A$. Then $u$ is an eigenfunction corresponding to the third eigenvalue and so $\operatorname{Index}(L) \geq 2$, a contradiction. In the case of a general Riemannian surface, $u$ may have only two zeros and then we have $\operatorname{Index}(L) \geq 1$ if $u$ is not identically zero.

\section{The Gauss-Codazzi equation of the generalized Riemann examples}

In this section we construct the family of Riemann examples in $M \times \mathbb{R}$, with $K_{M}=$ $c_{0}$ a constant. We classify all examples foliated by curves of constant curvature in the horizontal plane. These surfaces are annuli or simply connected surfaces transverse to each horizontal plane. We describe the space moduli of these surfaces in terms of elliptic functions.

We parametrize these surfaces by the third coordinate; in the notation of the previous section, the embedding $X=(F, y): \tilde{A}=\{(x, y) \in] \alpha_{1}, \alpha_{2}[\times] \beta_{1}, \beta_{2}[\} \rightarrow$ $M \times \mathbb{R}$ is minimal with $F: \tilde{A} \rightarrow M$ harmonic. Here $\tilde{A}$ is the universal covering of $A$ and $\alpha_{1}, \alpha_{2}, \beta_{1}, \beta_{2}$ can be infinite. We describe the space of these surfaces in terms of elliptic functions.

Let $\omega: \tilde{A} \rightarrow \mathbb{R}$ be the function defined by $d s^{2}=\cosh ^{2} \omega|d z|^{2}$. When $A$ is transverse to each horizontal plane, $\omega$ is finite on $A$ and is solution of the system

$$
\begin{aligned}
\triangle_{0} \omega+K_{M} \sinh \omega \cosh \omega & =0, \\
\omega_{x y}-\tanh \omega \omega_{x} \omega_{y} & =0 .
\end{aligned}
$$

From Proposition 2.1, we see that the first equation reflects the Gauss equation of $M$; the second states that of each level curve has constant curvature. In the case $K_{M}=1$, this system has been studied by Abresch [1987], who classified constant mean curvature tori in $\mathbb{R}^{3}$ with planar large lines of curvature (the second equation is the torsion of a large line of curvature of constant mean curvature surfaces).

To construct examples, we apply Abresch's technique in Theorem 3.1 to solve the system (7) on the whole plane $\mathbb{R}^{2}$. We will represent the space of these examples by a two parameter family. When a solution $\omega$ is periodic in the variable $x$ and $\omega \neq \infty$, we can expect an annulus by closing periods of the immersion. The 
harmonic map has to be periodic in $x$ and the immersion $X$ is well defined on $A=\left\{(x, y) \in \mathbb{R} /\left(x_{0} \mathbb{R}\right) \times \mathbb{R}\right\}$ (see Section 4).

A solution can take infinite values and then it will define the domain of $\tilde{A}$ where the solutions are well defined. When $c_{0}<0$, the condition $\omega \neq \infty$ is valid only in domains homeomorphic to a strip, a disk or the plane with a countable set of disks removed. In particular there are helicoidal surfaces embedded in $\mathbb{H}^{2} \times \mathbb{R}$ defined on a strip. The set where $\omega$ is infinite represents a curve in the boundary at infinity, $\partial_{\infty} \mathbb{H} \mathbb{Z}^{2} \times \mathbb{R}$.

Using these solutions $\omega$, we use Gauss-Codazzi equation to construct a harmonic map $F: \tilde{A} \rightarrow M$ in Theorem 3.2. It remains to study the period problem and the geometry of the family in Section 4.

Theorem 3.1. Let $\omega: \mathbb{R}^{2} \rightarrow \mathbb{R}$ be a real-analytic solution of the system (7), with $K_{M}=c_{0}$ a given constant. We define $f, g$ as functions of $\omega$ by

$$
f=\frac{-\omega_{x}}{\cosh \omega} \quad \text { and } \quad g=\frac{-\omega_{y}}{\cosh \omega}
$$

Then the real functions $x \mapsto f(x)$ and $y \mapsto g(y)$ of one variable solve the system

$$
\begin{aligned}
-\left(f_{x}\right)^{2} & =f^{4}+\left(c_{0}+a\right) f^{2}+c, \\
-f_{x x} & =2 f^{3}+\left(c_{0}+a\right) f \quad \text { with } c, d \in \mathbb{R}, a=\frac{c-d}{c_{0}} \text { if } c_{0} \neq 0, \\
-\left(g_{y}\right)^{2} & =g^{4}+\left(c_{0}-a\right) g^{2}+d \quad \text { with } c=d \text { and } a \in \mathbb{R} \text { if } c_{0}=0, \\
-g_{y y} & =2 g^{3}+\left(c_{0}-a\right) g .
\end{aligned}
$$

Conversely, we can recover the solution $\omega$ from $f$ and $g$. If $c_{0}+f^{2}+g^{2}$ is not identically zero we have

$$
\sinh \omega=\left(c_{0}+f^{2}+g^{2}\right)^{-1}\left(f_{x}+g_{y}\right)=\left(f_{x}-g_{y}\right)^{-1}\left(g^{2}-f^{2}-a\right) .
$$

If $c_{0}+f^{2}+g^{2} \equiv 0$ on $\mathbb{R}^{2}$, the functions $f:=\alpha$ and $g:=\beta$ are constant and the solutions are given by

$$
\sinh \omega=-\tan (\alpha x+\beta y) .
$$

When $c_{0} \leq 0$, the solution $\omega$ may have infinite values. Setting $D=\left\{(x, y) \in \mathbb{R}^{2}\right.$ : $\omega=\infty\}$ we have, in the case of equation (9),

$$
\begin{aligned}
& D=\left\{(x, y) \in \mathbb{R}^{2}: f^{2}+g^{2}+c_{0}=0 \text { and } f_{x}-g_{y}=0\right\} \text { for } c \neq 0, d \neq 0, \\
& D=\left\{(x, y) \in \mathbb{R}^{2}: f^{2}+c_{0}=0\right\} \text { for } d=0, \\
& D=\left\{(x, y) \in \mathbb{R}^{2}: g^{2}+c_{0}=0\right\} \text { for } c=0,
\end{aligned}
$$


and in the case where $\omega$ is given by equation (10),

$$
D=\left\{(x, y) \in \mathbb{R}^{2}: \alpha x+\beta y=\frac{1}{2} k \pi, k \in \mathbb{Z}\right\} .
$$

When $c_{0}$ vanishes, $D$ is a countable set of isolated points $(x, y)$, namely those where $f(x)=g(y)=0$.

When $c_{0}>0$, there is a solution of the system if and only if $c \leq 0$ and $d \leq 0$ and $\omega$ is periodic and defined on the whole plane $\mathbb{R}^{2}$.

Proof. We apply Abresch's technique with $K_{M}=c_{0}$ a given constant. Let $\omega$ be a solution of (7). We work at the point where $c_{0}+f^{2}+g^{2} \neq 0$. The second equation of (7) leads to separation of the variables:

$$
\cosh \omega f_{y}=\tanh \omega \omega_{x} \omega_{y}-\omega_{x y}=\cosh \omega g_{x}=0 .
$$

Then $\omega$ solves (7) if and only if $f$ and $g$ depend on one variable and satisfy the equation

$$
f_{x}+g_{y}=\frac{-\triangle_{0} \omega}{\cosh \omega}+\tanh \omega \frac{\omega_{x}^{2}+\omega_{y}^{2}}{\cosh \omega}=\left(c_{0}+f^{2}+g^{2}\right) \sinh \omega
$$

Now we integrate $f, g$. Differentiating and substituting, we get

$$
\frac{f_{x x}}{c_{0}+f^{2}+g^{2}}-f \frac{f_{x}^{2}-g_{y}^{2}}{\left(c_{0}+f^{2}+g^{2}\right)^{2}}=-f
$$

and

$$
\frac{g_{y y}}{c_{0}+f^{2}+g^{2}}-g \frac{g_{y}^{2}-f_{x}^{2}}{\left(c_{0}+f^{2}+g^{2}\right)^{2}}=-g .
$$

Multiplying (13) by $2 f_{x}$ and integrating with respect to $x$, we obtain, for a constant $k(y)$,

$$
\frac{f_{x}^{2}-g_{y}^{2}}{c_{0}+f^{2}+g^{2}}=-f^{2}+k(y) .
$$

Multiplying (13) and (14) by $\left(c_{0}+f^{2}+g^{2}\right)$ and $f$ respectively, and adding them together, we get

$$
f_{x x}=-2 f^{3}-\left(c_{0}+g^{2}(y)-k(y)\right) f .
$$

Since $f$ does not depend on $y$, we can pick any of the values of $c_{0}+g^{2}+k(t)$ for $\bar{c}$ and get

$$
-f_{x x}=2 f^{3}+\bar{c} f .
$$

A similar computation holds for $g$, yielding

$$
-g_{y y}=2 g^{3}+\bar{d} g
$$


These equations have first integrals

$$
-\left(f_{x}\right)^{2}=f^{4}+\bar{c} f^{2}+c, \quad-\left(g_{y}\right)^{2}=g^{4}+\bar{d} g^{2}+d .
$$

If we take real functions $f, g$ satisfying equations (15)-(17), we get a function $\omega$ by (9). Now $\omega$ is defined and solves the system (7) if and only if $f$ and $g$ can be expressed as in (8). By taking the derivative in (9), one can prove that (8) is equivalent to (13). We plug (15)-(17) into (13) to conclude that $\omega$ is a solution of (12) if and only if

$$
\begin{aligned}
& f\left(c_{0}^{2}-\bar{c} c_{0}+c-d\right)+f g^{2}\left(2 c_{0}-\bar{c}-\bar{d}\right)=0, \\
& g\left(c_{0}^{2}-\bar{d} c_{0}+d-c\right)+f^{2} g\left(2 c_{0}-\bar{d}-\bar{c}\right)=0 .
\end{aligned}
$$

Then for $c_{0} \neq 0$, if $f \neq 0$ and $g \neq 0$, we deduce from (18) that

$$
\bar{c}=c_{0}+\frac{c-d}{c_{0}}=c_{0}+a \quad \text { and } \quad \bar{d}=2 c_{0}-\bar{c}=c_{0}-a .
$$

If $f \equiv 0$ and $g \neq 0$, we have $c=0$ and from (19) we derive $\bar{d}=c_{0}+\frac{d}{c_{0}}=c_{0}-a$, while if $g \equiv 0$ and $f \neq 0, d=0$ and

$$
\bar{c}=c_{0}+\frac{c}{c_{0}}=c_{0}+a .
$$

When $c_{0}=0$, if $f \neq 0, g \neq 0$ we have $c=d$ and $\bar{c}=-\bar{d}$; if $f=0$ or $g=0$ then $c=d=0$.

All our computations are valid at points where $c_{0}+f^{2}+g^{2} \neq 0$, but $f$ and $g$ are real functions defined on $\mathbb{R}$. At a point where $c_{0}+f^{2}+g^{2}=0$, one can consider the value of $\omega$ by passing to the limit in (9). It depends on $f_{x}+g_{y}$. We note that

$$
f_{x}^{2}-g_{y}^{2}=\left(c_{0}+g^{2}+f^{2}\right)\left(g^{2}-f^{2}-a\right)
$$

and that $\omega$ is well defined if $f_{x}+g_{y}=0$ and $f_{x}-g_{y} \neq 0$ (that is, $f_{x}=-g_{y} \neq 0$ ). Then we can define $\omega$ by continuity at this point.

If $f_{x}+g_{y} \neq 0$ and $f_{x}-g_{y}=0$, then $\omega$ has a pole.

In the case where $f_{x}=g_{y}=0$ and $c_{0}+f^{2}+g^{2}$ vanishes at a point, we have $f_{x x}=g_{y y}=0$ if and only if $g^{2}-f^{2}-a=0$ by differentiation of (12), and then $f$ and $g$ are constant by the unique continuation theorem. Otherwise, if $f_{x}=g_{y}=0$, $c_{0}+f^{2}+g^{2}=0$, and $g^{2}-f^{2}-a \neq 0$, again $\omega$ has a pole. Thus $D=\{(x, y) \in$ $\mathbb{R}^{2}: c_{0}+f^{2}+g^{2}=0$ and $\left.f_{x}-g_{y}=0\right\}$ when $f$ and $g$ are not identically constant.

In the case where $c_{0}+f^{2}+g^{2} \equiv 0$ and $f_{x}=g_{y} \equiv 0$ we have $f^{2}=\frac{1}{2}(1+c-d)=$ : $\alpha^{2}$ and $g^{2}=\frac{1}{2}(1+d-c)=: \beta^{2}$, with the additional condition $(1+d-c)^{2}=4 d$. In this case, one can integrate directly the solutions and then $\sinh \omega=-\tan (\alpha x+\beta y)$. 
In the case where $d=0$, we have $g \equiv 0$ and $-\left(f_{x}\right)^{2}=\left(f^{2}-c\right)\left(f^{2}+c_{0}\right)$. If $c=1$, we have $f \equiv 1$. Otherwise

$$
\sinh \omega=\frac{f_{x}}{f^{2}+c_{0}}=\frac{f^{2}-c}{-f_{x}} \rightarrow \infty \quad \text { as } f^{2} \rightarrow-c_{0} \quad\left(\text { and then } f_{x} \rightarrow 0\right),
$$

which proves that $\omega=\infty$ on $D=\left\{(x, y) \in \mathbb{R}^{2}: f^{2}+c_{0}=0\right\}$. The same is true for $c=0$.

When $c_{0} \geq 0$, the functions $f$ and $g$ are defined if and only if $c \leq 0$ and $d \leq 0$. To see this, notice that the values of $f^{2}$ and $g^{2}$ lie between distinct zeroes of $X^{2}+\left(c_{0}+a\right) X+c$ and $Y^{2}+\left(c_{0}-a\right) Y+d$ respectively. Assume $c>0$; then $0 \leq\left(c_{0}+a\right)^{2}-4 c<\left(c_{0}+a\right)^{2}$. In the case $\left(c_{0}+a\right)>0$ we find

$$
-\left(c_{0}+a\right)-\sqrt{\left(c_{0}+a\right)^{2}-4 c} \leq 2 f^{2} \leq-\left(c_{0}+a\right)+\sqrt{\left(c_{0}+a\right)^{2}-4 c}<0,
$$

and if $\left(c_{0}+a\right)<0$, we have $c_{0}-a>2 c_{0}>0$ and $0<c<d$, hence

$$
-\left(c_{0}-a\right)-\sqrt{\left(c_{0}-a\right)^{2}-4 c} \leq 2 g^{2} \leq-\left(c_{0}-a\right)+\sqrt{\left(c_{0}-a\right)^{2}-4 c}<0 .
$$

This contradicts the fact that $g$ is a real valued function. We conclude that $c \leq 0$ and similarly $d \leq 0$.

We now use the Gauss-Codazzi equations to integrate solutions of (7).

Theorem 3.2. Let $\omega$ be a solution of the system (7) on a simply connected domain $\Omega$, then there exists a minimal isometric embedding of $\left(\Omega, d s^{2}=\cosh ^{2} \omega|d z|^{2}\right)$ in $M\left(c_{0}\right) \times \mathbb{R}$ foliated by constant curvature curves at each horizontal level.

Proof. Let $\omega$ be a solution of (7). When $c_{0}>0, \omega$ is defined on the whole plane. For $c_{0}=1$, it is a well-known fact that the first equation is the Gauss condition of local existence of a constant mean curvature surface $H=1 / 2$ in $\mathbb{R}^{3}$ (it is a sinh-Gordon equation; see [Abresch 1987]). Since $\Omega$ is simply connected, there is a constant mean curvature immersion $H: \Omega \rightarrow \mathbb{R}^{3}$, and its Gauss map $F: \Omega \rightarrow \mathbb{S}^{2}$ is the harmonic map associated with $\omega$ [Abresch 1987].

In the case $c_{0}=-1$, one can use the same construction explained in [Wan 1992] or [Akutagawa and Nishikawa 1990]. The system (7) gives us a Gauss equation to construct a space-like surface of constant mean curvature in the Minkowski space $M^{2,1}$ with Hopf map $Q=\frac{1}{4}(d z)^{2}$. The unit normal vector to this surface in $M^{2,1}$ is a harmonic map $F: \Omega \rightarrow \mathbb{H}^{2}$ associated with the solution $\omega$.

For $c_{0} \neq 0$, we use a dilatation. Consider

$$
\widetilde{\omega}=\omega\left(\frac{x}{\sqrt{\left|c_{0}\right|}}, \frac{y}{\sqrt{\left|c_{0}\right|}}\right)
$$

which is a solution of $\triangle_{0} \widetilde{\omega}=\left(c_{0} /\left|c_{0}\right|\right) \sinh \widetilde{\omega} \cosh \widetilde{\omega}$. Then we find a harmonic 
$\operatorname{map} \widetilde{F}: \Omega\left(\sqrt{\left|c_{0}\right|}\right) \rightarrow M( \pm 1)$ with

$$
\Omega\left(\sqrt{\left|c_{0}\right|}\right)=\left\{(x, y) \in \mathbb{R}^{2}:\left(\frac{x}{\sqrt{\left|c_{0}\right|}}, \frac{y}{\sqrt{\left|c_{0}\right|}}\right) \in \Omega\right\} .
$$

Now with a dilatation, we have the harmonic map

$$
F=\left|c_{0}\right| \widetilde{F}\left(\sqrt{\left|c_{0}\right|} x, \sqrt{\left|c_{0}\right|} y\right): \Omega \rightarrow M\left(c_{0}\right),
$$

which corresponds to our system (7).

Immersions are given by $X=(F, y)$ on $\Omega$. The second equation in (7) states that these examples are foliated by constant curvature curves at each horizontal level (see Proposition 2.1).

For minimal surfaces in $\mathbb{R}^{3}$, we construct a surface by considering the Weierstrass data $g=-i e^{\omega+i \psi}$ and $\eta=-i d z$, where $\omega+i \psi$ is holomorphic.

$$
2 X(z)=\operatorname{Re} \int_{z}\left(\left(g^{-1}-g\right) \eta, i\left(g^{-1}+g\right) \eta, 2 \eta\right) .
$$

Now we describe some geometric properties of these families of surfaces. Let $\omega$ be a solution of (7) on $\tilde{A}$ described in Theorem 3.2, then $X=(F, y)$ is a minimal surface foliated by horizontal curves of constant curvature. By Proposition 2.1, $g(y)$ is the curvature of $A \cap M \times\{y\}$ and $-f\left(x_{0}\right) \operatorname{coth} \omega$ is the geodesic curvature of the projection of $X\left(x_{0}, y\right)$ on $M \times\{0\}$. We will consider $c_{0}=+1,0,-1$ as generic cases; other cases come from dilatation. We describe the geometry of examples in these space forms.

\section{The geometry of generalized Riemann examples}

\subsection{Minimal surfaces in $\mathbb{S}^{2} \times \mathbb{R}$.}

Theorem 4.1. The space of minimal surfaces of genus zero embedded in $\mathbb{S}^{2} \times \mathbb{R}$ and foliated by horizontal curves of constant curvature is a two-parameter family parametrized by $\mathcal{M}=\left\{(c, d) \in \mathbb{R}^{2}: c \leq 0, d \leq 0\right\}$. All examples are annuli periodic in the vertical direction.

(i) $c=0, d \in \mathbb{R}_{-}$is a family unduloids (surfaces of revolution described in [Pedrosa and Ritoré 1999] and [Rosenberg 2002]). The curvature of the horizontal curves oscillates between values of opposite sign.

(ii) $d=0, c \in \mathbb{R}_{-}$is a family of helicoids. The curves of horizontal constant curvature are geodesics passing through two antipodal points (the axis).

(iii) $(c, d) \in\left(\mathbb{R}_{-}^{*}\right)^{2}$ is a two-parameter family of Riemann type surfaces. These annuli are foliated by circles whose radius oscillates between two opposite values and whose center are located on a given geodesic.

(iv) $(c, d)=(0,0)$ is a vertical flat annulus foliated by a great circle. 
Proof. In the case where $K_{M}=c_{0}>0$ (i.e., $M=\mathbb{S}^{2}$ up to a homothety in $\mathbb{R}^{4}$ ), the functions $f, g$ are described as in [Abresch 1987], where it is shows that they are both periodic, oscillating around zero. The zeros of $X^{2}+\left(c_{0}+a\right) X+c$ and $Y^{2}+\left(c_{0}-a\right) Y+d$ have opposite signs and then $f^{2} \in\left[0, X_{+}\right], g^{2} \in\left[0, Y_{+}\right]$. Assume that $f(0)=0$ and $g(0)=0, f_{x}(0)=\alpha \geq 0, g_{y}(0)=\beta \geq 0$ with $c=-\alpha^{2}$ and $d=-\beta^{2}$. By Proposition 2.1, $g$ is the curvature of horizontal curves (it does not depend on $x)$ while $-f(v) \operatorname{coth} \omega$ corresponds to the curvature of the curve $\gamma_{v}$ obtained by projection of $X(v, y)$ onto $\mathbb{S}^{2} \times\{0\}$. The tangent vector of this curve is $F_{y}$ and $\left\langle F_{y}, F_{x}\right\rangle$ vanishes; thus if $(v, y)$ is chosen such that $f(v)=0$ and $\omega(v, y) \neq 0$, the curve $\gamma_{v}$ is a geodesic orthogonal to each horizontal level curve. When $f(v)=0$ and $\omega(v, y)=0$, the tangent vector $F_{y}$ vanishes and the corresponding curve is a vertical straight line.

Since curves of constant curvature are periodic on $\mathbb{S}^{2}$, the immersion of $\tilde{A}$ is the covering of a minimal annulus embedded in $\mathbb{S}^{2} \times \mathbb{R}$. From these facts we deduce the following.

When $d=0$ and $c \leq 0$, the horizontal curves are geodesics (great circles in $\mathbb{S}^{2}$ ) and $f_{x}$ has two zeroes, $x_{0}$ and $x_{1}$. Then $\sinh \omega\left(x_{0}, y\right)=\sinh \omega\left(x_{1}, y\right)=0$, which corresponds to two vertical axes at antipodal points $F_{y}\left(x_{0}, y\right)=F_{y}\left(x_{1}, y\right)=0$. This is the helicoidal family described in [Rosenberg 2002].

When $c=0$ and $d \leq 0$, the horizontal curves have constant geodesic curvature oscillating between $d$ and $-d$. The function $f=0$ and $\omega \neq 0$ if $g \neq 0$. Then the center of each horizontal circle is at the same point. It is a rotational invariant surface. It is the unduloid family of Pedrosa and Ritoré [1999], also described in [Rosenberg 2002].

The other surfaces ( $c<0$ and $d<0$ ) are of Riemann type in the following sense. The horizontal curves are circles whose radius oscillates periodically between two values of opposite sign. In particular there are horizontal geodesics in the surface, at periodic intervals. The center of each circle is located on a fixed geodesic by taking the geodesic described by $\gamma_{v}$ above with $f(v)=0$.

\subsection{Minimal surfaces in $\mathbb{U}^{2} \times \mathbb{R}$.}

Theorem 4.2. The space of minimal surfaces of genus zero embedded in $\mathbb{H}^{2} \times \mathbb{R}$ and foliated by horizontal constant curvature curves is a two parameter family parametrized by (see Figure 1)

$$
\mathcal{M}=\left\{(c, d) \in \mathbb{R}^{2}:(1+c-d)^{2} \geq 4 c \text { and } c-1 \leq d \leq c+1\right\} \cup\{c \leq 0\} \cup\{d \leq 0\} .
$$

We first describe some special one-parameter families of $M$ :

(1) The curve $\Gamma=\left\{(c, d) \in \mathbb{R}^{2}:(1+c-d)^{2}=4 c\right.$ and $\left.c-1 \leq d \leq c+1\right\}$ parametrizes surfaces of helicoidal type where the horizontal curves have 


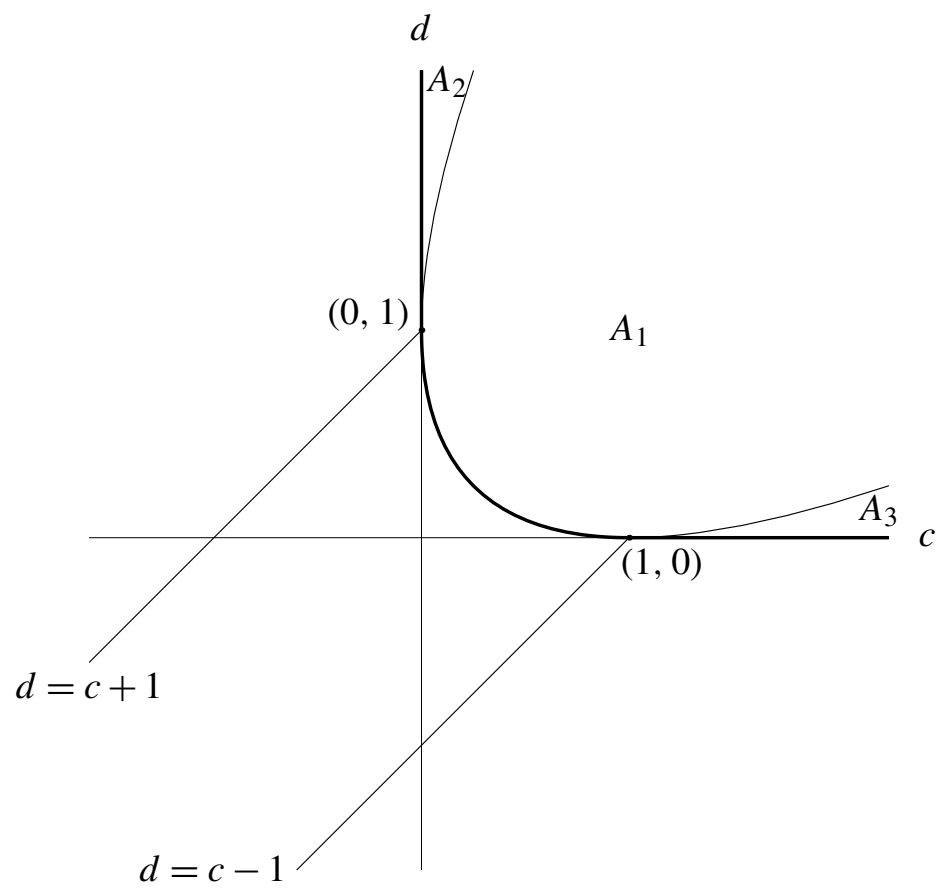

Figure 1. Parameter space of Theorem 4.2. $\mathcal{M}$ is the complement of $A_{1} \cup A_{2} \cup A_{3}$ (below and to the left of the thick lines).

constant curvature $k=g=\frac{1}{2} \sqrt{1+d-c}$. When $(c, d)=(1,0)$ the surface is a helicoid. When $(c, d)=(0,1)$ it is an annulus foliated by horocycles.

(2) $d=0$. The surfaces are foliated by horizontal geodesics.

(a) Values $c>0$ parametrize the helicoidal family.

(b) For $c<0$ the surfaces are global graphs on $\mathbb{Q}^{2}$ and can be likened to oblique planes.

(c) When $(c, d)=(0,0)$, the surface is a vertical geodesic plane $\gamma \times \mathbb{R}$.

(3) $c=0$. The surfaces are bounded in the third component. They are catenoids and graphs.

(a) $d>1$. The surfaces are rotational annuli related to catenoidal examples. (They are described in [Nelli and Rosenberg 2002].)

(b) $0<d \leq 1$. The examples are catenoids foliated by equidistant curves in $\mathbb{H}^{2}$ (curves with curvature $0<k<1$, equidistant to a geodesic). In a euclidean sense, the surface is homeomorphic to a part of a catenoid described in (3a), intersecting a solid cylinder with axis translated by a horizontal translation, in such a way that every horizontal circle intersects the boundary of the cylinder.

(c) $d<0$. The surface is a global graph on $\mathbb{H}^{2}$, foliated by equidistant curves. 

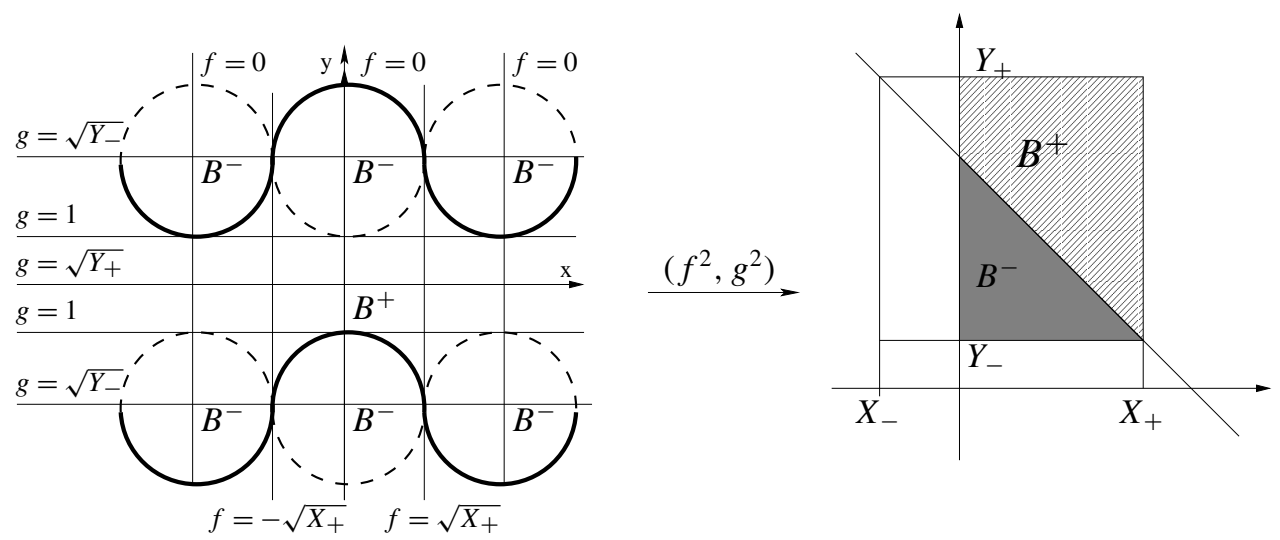

$$
\text { - - } B \cap\left\{(x, y) \in \mathbb{R}^{2} ; f_{x}+g_{y}=0\right\}
$$

$\longrightarrow D=\left\{(x, y) \in \mathbb{R}^{2} ; \omega= \pm \infty\right\}=B \cap\left\{(x, y) \in \mathbb{R}^{2} ; f_{x}-g_{y}=0\right\}$

Figure 2. The annulus family: surface parametrization (top) and schematic view in $\mathbb{H}^{2} \times \mathbb{R}$ (right).

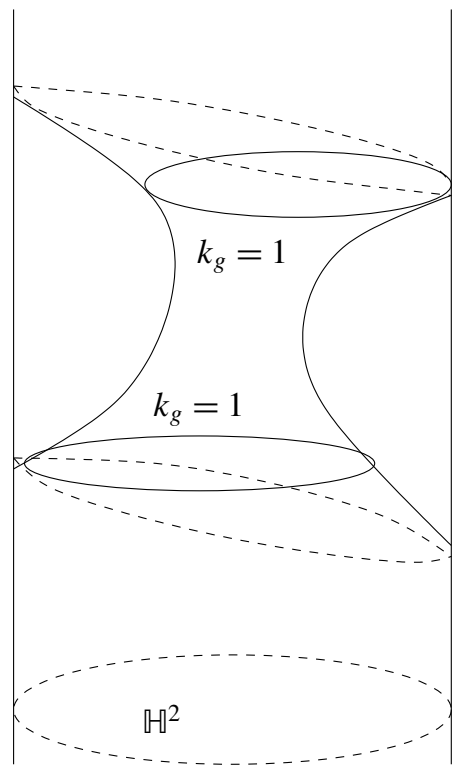

Now we describe the regions of $M$ bounded by curves described in (1)-(3).

(4) Second quadrant $(c<0$ and $d>0)$. The surfaces are annuli with two nonhorizontal boundary curves at infinity. These annuli contain two horocycles in some horizontal section. They are parametrized in a region homeomorphic to a strip. (See Figure 2.)

(5) Fourth quadrant ( $c>0$ and $d<0$ ). The surfaces are "undulated helicoids". They are parametrized by a vertical strip and are periodic in the third component (see Figure 3). In a period there are two horizontal geodesics in the 


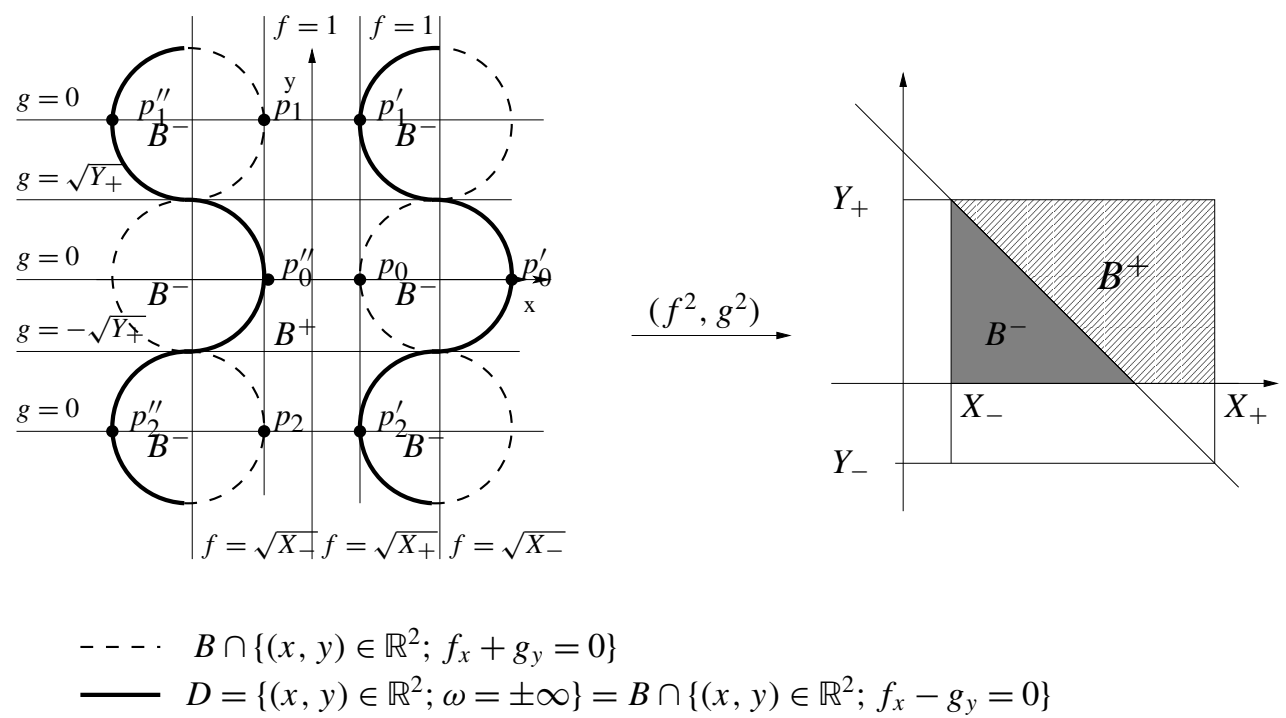

Figure 3. The helicoidal family of the fourth quadrant.

surface. Between these two horizontal sections, the surface is foliated by equidistant curves $(k<1)$. The surface is undulated in the sense that the curvature changes sign after crossing a geodesic.

(6) First quadrant ( $c>0$ and $d>0)$. The surfaces are "blown-up helicoids". They are parametrized by a strip (see Figure 4) but the curvature of the horizontal curves is never zero.

(7) Third quadrant $(c<0$ and $d<0)$. The surfaces are Riemann type examples. They are parametrized conformally by a cylinder minus a countable set of disk (see Figure 5). They have a vertical plane of symmetry and the boundary set of curves at infinity is a disjoint set of circles in the cylinder (see Figure 6).

Remark. Daniel [2004] gives explicit formulas for the surfaces in (1)-(3).

Proof. In the case where $K_{M}=-1$ (we can consider $\mathbb{H}^{2}$ as the universal covering of $M$ ), the family is quite important. The existence of solutions $f$ and $g$ depends on $P(X)=X^{2}-(1-a) X+c=X^{2}-(1+c-d) X+c$ and $Q(y)=Y^{2}-(1+a) Y+d=$ $Y^{2}-(1+d-c) Y+d$. Note that $P, Q$ have same the discriminant,

$$
\Delta=(1+c-d)^{2}-4 c=(1+d-c)^{2}-4 d .
$$

The roots of $P(X)=0$ are

$$
X_{+}=\frac{1}{2}(1+c-d+\sqrt{\Delta}) \quad \text { and } \quad X_{-}=\frac{1}{2}(1+c-d-\sqrt{\Delta})
$$




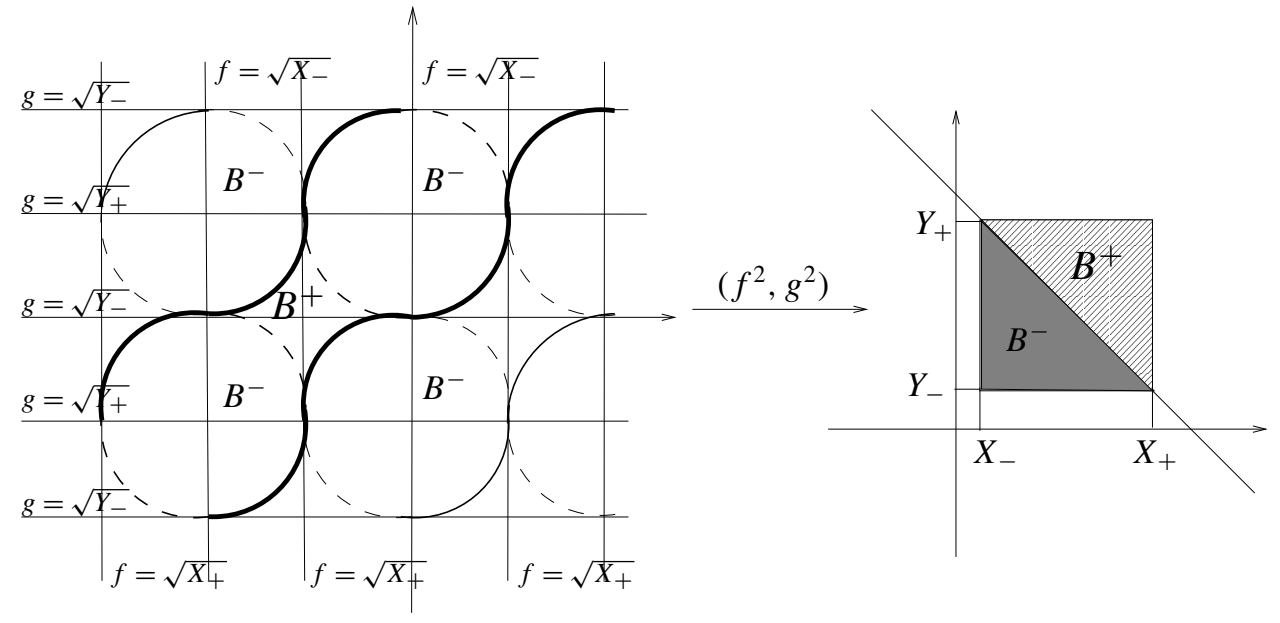

$$
\begin{array}{ll}
\longrightarrow & B \cap\left\{(x, y) \in \mathbb{R}^{2} ; f_{x}+g_{y}=0\right\} \\
& D=\left\{(x, y) \in \mathbb{R}^{2} ; \omega= \pm \infty\right\}=B \cap\left\{(x, y) \in \mathbb{R}^{2} ; f_{x}-g_{y}=0\right\}
\end{array}
$$

Figure 4. The helicoidal family of the first quadrant.

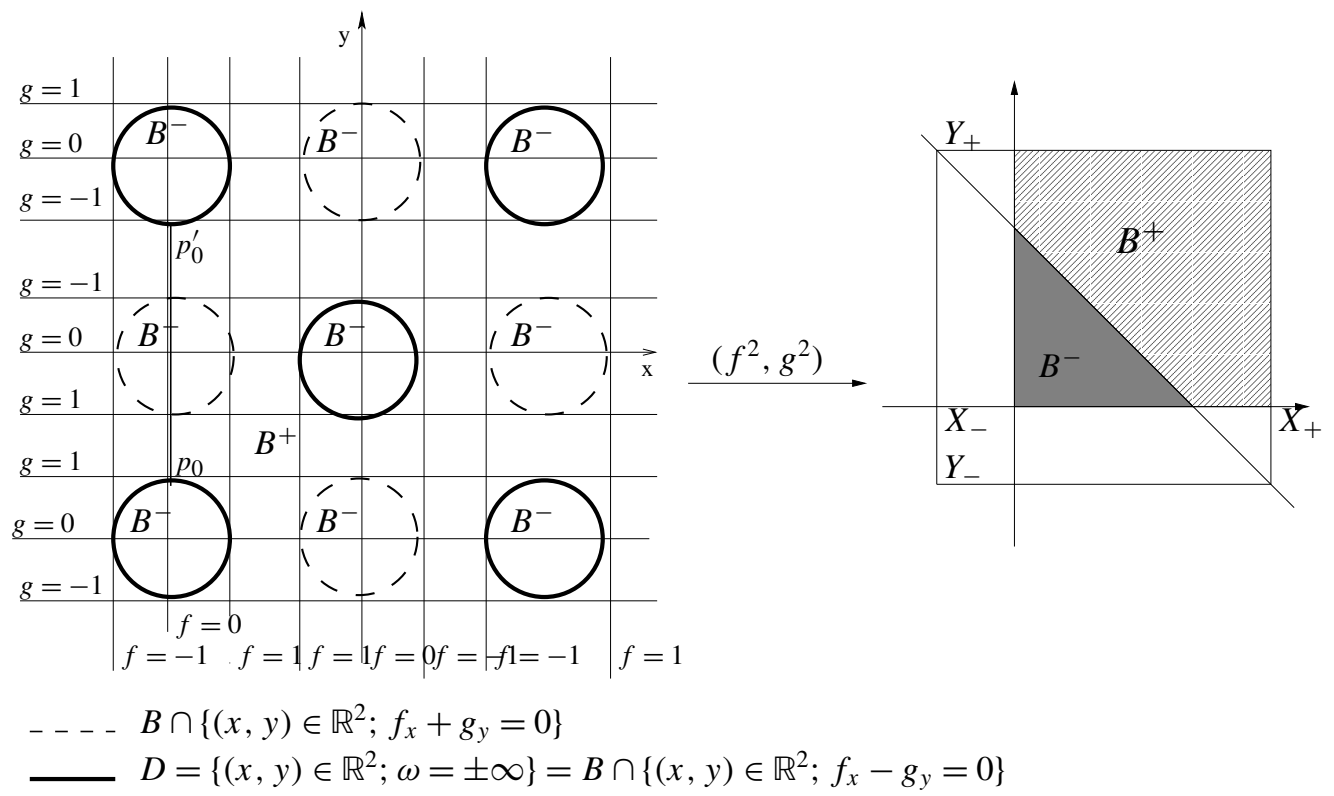

Figure 5. The Riemann family.

and those of $Q(Y)=0$ are

$$
Y_{+}=\frac{1}{2}(1+d-c+\sqrt{\Delta}) \quad \text { and } \quad Y_{-}=\frac{1}{2}(1+d-c-\sqrt{\Delta}) .
$$



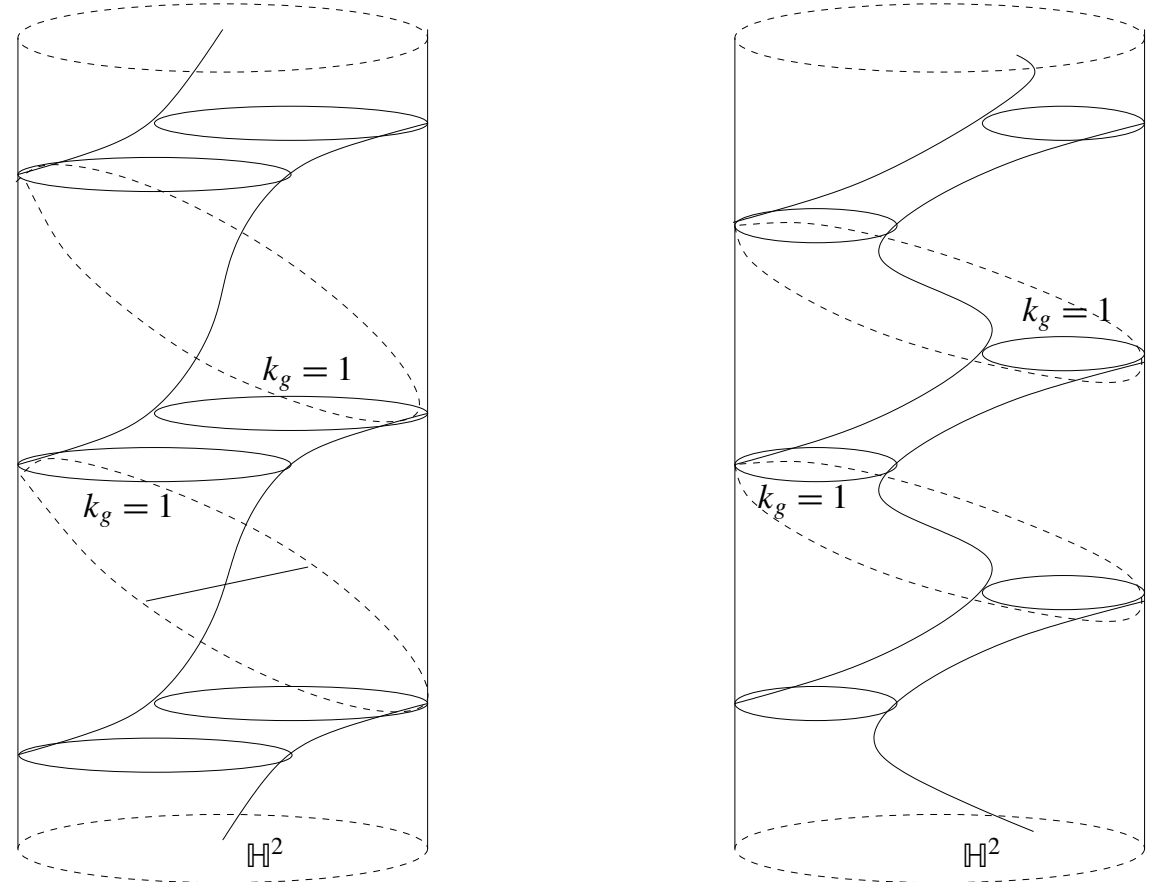

Figure 6. Two examples of Riemann type in $\mathbb{U}^{2} \times \mathbb{R}$.

Since $P\left(f^{2}\right)=-\left(f_{x}\right)^{2}$ and $Q\left(g^{2}\right)=-\left(g_{y}\right)^{2}$, the functions $f$ and $g$ exist if and only if $\Delta \geq 0$ and $X_{+} \geq 0, Y_{+} \geq 0$. We parametrize our family of surfaces in the plane $(c, d)$ in Figure 1 .

When $\Delta=0$, the functions $f^{2}$ and $g^{2}$ are constant; then $f_{x}$ and $g_{y}$ vanish and $\omega$ is given by (10). We can see that $\Delta<0$ if and only if $(c+d)>\frac{1}{2}(c-d)^{2}+\frac{1}{2}$, that is, in the convex region $A_{1}$ bounded by the parabola in Figure 1.

Moreover one can see that when $d>c+1, c>0$ and $\Delta>0$ (region $A_{2}$ ), we have $1+c-d \leq-\sqrt{\Delta}<0$ and then $X_{+}<0$. In the case $c>d+1, d>0$ and $\Delta>0$ (region $A_{3}$ ), we have $Y_{+}<0$. Then

$$
\mathcal{M}=\left\{(c, d) \in \mathbb{R}^{2}: \Delta \geq 0, X_{+} \geq 0, Y_{+} \geq 0\right\}=\mathbb{R}^{2}-\left(A_{1} \cup A_{2} \cup A_{3}\right) .
$$

The important phenomenon here is that $f^{2}+g^{2}-1$ can be zero. To help us in the following we define

$$
\begin{aligned}
& B=\left\{(x, y) \in R^{2}: f^{2}+g^{2}=1\right\}, \\
& B^{-}=\left\{(x, y) \in R^{2}: f^{2}+g^{2}<1\right\}, \\
& B^{+}=\left\{(x, y) \in R^{2}: f^{2}+g^{2}>1\right\} .
\end{aligned}
$$


Now the set $D=B \cap\left\{(x, y) \in R^{2}: f_{x}-g_{y}=0\right\}$ separates $\mathbb{R}^{2}$ in connected components where our surfaces are defined.

In the second quadrant ( $c<0$ and $d>0$ ) we have an annulus bounded in the third component. In the fourth $(c>0$ and $d<0)$ and first $(c>0$ and $d>0)$ quadrants the surfaces are homeomorphic to simply connected strips embedded in the cylinder. In the third quadrant $(c<0$ and $d<0)$ we have minimal surfaces of Riemann type; $\mathbb{R}^{2}-D$ is the plane with a countable set of disks removed.

First we classify the examples depending only on one parameter, $x$ or $y$.

(1) Recall that $\Gamma$ is defined as the set of points $(c, d)$ such that $(1+c-d)^{2}=4 c$ (that is, $\Delta=0$ ) and $c-1 \leq d \leq c+1$. On $\Gamma$ we have and $X_{+}=X_{-}, Y_{+}=Y_{-}$, so $f_{x}=g_{y}=0$ and $f^{2}+g^{2}=1$. We are in the case where $\omega$ is given by formula (10). The surface is foliated by curves of constant geodesic curvature $k=g=$ $\sqrt{(1+d-c) / 2}=: \alpha \leq 1$. By (10), $\omega \neq \infty$ if and only if $-\pi / 2<\alpha x+\beta y<\pi / 2$. Then $\omega$ is defined on a strip and the straight line $\alpha x+\beta y=0$ defines an axis, since $\omega=0$. After an isometry, we can assume that the axis projects to the origin of the Poincare disk model. The horizontal vector $F_{x}$ has argument $\psi$, which in view of (5) and the equality $\omega(-(\beta / \alpha) y, y)=0$ has derivative $\psi_{y}=1 / \alpha$ (use $\rho(0)=1$ and $\rho_{u_{1}}(0)=\rho_{u_{2}}(0)=0$ in (5)). The horizontal curves turn with constant speed. The case $c=0$ is parametrized on a horizontal strip. The third component is bounded and the horizontal curves are all horocycles $\left(k_{g}=1\right)$. The horizontal section $\{y=0\}$ is a plane of symmetry of this annulus.

(2) The helicoid and planar family $d=0$. First, we classify the family of surfaces foliated by geodesics at each horizontal level ( $g=0$ identically and $d=0)$. In this case we have $-\left(f_{x}\right)^{2}=f^{4}-(1+c) f^{2}+c=\left(f^{2}-c\right)\left(f^{2}-1\right)$. The case $c=0$ and $d=0$ represents a geodesic vertical plane $\gamma \times \mathbb{R}$ (a geodesic product of $\mathbb{R}$ and $\omega=0)$. For $c \neq 0$ we have

$$
\sinh \omega=\frac{f_{x}^{2}}{f_{x}\left(f^{2}-1\right)}=\frac{-\left(f^{2}-c\right)}{f_{x}} \rightarrow \pm \infty \quad \text { as } f^{2} \rightarrow 1 .
$$

Then the surface is defined on the vertical strip $D$ bounded by the set $D=\{(x, y) \in$ $\mathbb{R}^{2}: x=a_{0}$ and $\left.x=a_{1}\right\}$ (with $f^{2}\left(a_{0}\right)=f^{2}\left(a_{1}\right)=1$ ). Each component of the complement $\mathbb{R}^{2}-\left\{f^{2}=1\right\}$ gives rise to the same surface.

We further distinguish two subcases:

(2a) $c>0$. The case $c=1$ is given in (1). If $c>1$, we have $B^{-}=\varnothing$ and the surface is parametrized on $B^{+}=\left\{(x, y) \in \mathbb{R}^{2}: a_{0}<x<a_{1}\right\}$. If $0<c<1$, the set $B^{+}$is empty and the surface is parametrized on $B^{-}=\left\{(x, y) \in \mathbb{R}^{2}: a_{0}<x<a_{1}\right\}$. When $f^{2}\left(x_{0}\right)=c$ the derivative $f_{x}$ vanishes and the locus $\sinh \omega=0$ describes a vertical axis. We can assume that $X\left(x_{0}, y\right)$ projects to the origin in the Poincare model of $\mathbb{M}^{2}$. For the other values of $f$, the projection consists of circles of curvature greater 
than 1 in the horizontal plane. These curves describe helicoidal movement in the cylinder model of $\mathbb{H}^{2} \times \mathbb{R}$. Note that horizontal geodesics (radii of the disc) turn with constant speed. The horizontal vector $F_{x}$ has argument $\psi$, which by the first equation (5) and the equality $\omega\left(x_{0}, y\right)=0$ has derivative $\psi_{y}\left(x_{0}, y\right)=\omega_{x}\left(x_{0}, y\right)=$ $-f\left(x_{0}\right)$ (a constant speed of rotation).

(2b) $c<0$. We have $-\left(f_{x}\right)=\left(f^{2}-c\right)\left(f^{2}-1\right)$ and then $f^{2} \in[0,1]$; that is, $f \in[-1,1]$ and the surface is parametrized by $B^{-}\left(B^{+}=\varnothing\right)$. We have $f_{x}$ of constant sign. We are looking for $k_{g}\left(\gamma_{v}\right)=-f(x) \operatorname{coth} \omega$. When $f\left(x_{0}\right)=0$, we have $\sinh \omega= \pm \sqrt{-c} \neq 0$ and $k_{g}\left(\gamma_{v}\right)=-f\left(x_{0}\right) \operatorname{coth} \omega=0$ but $\left|F_{y}\right| \neq 0$. Then the curve $X\left(x_{0}, y\right)$ projects on a geodesic of the disc which contains the center of the disc by assumption. The horizontal curves are geodesics crossing $X\left(x_{0}, y\right)$ in an orthogonal way (since $\left\langle F_{x}, F_{y}\right\rangle_{g}=0$ ). These surfaces are, so to speak, oblique planes in $\mathbb{M}^{2} \times \mathbb{R}$. As $c$ approaches 0 , these planes converge to a vertical plane (a geodesic $\times \mathbb{R}$ ) and when $c$ approaches $-\infty, f_{x}$ takes large values and these surfaces converge to a horizontal section.

(3) The catenoid family $c=0$. Now we classify the family of rotational type. The vertical curves $X\left(x_{0}, y\right)$ project to geodesics on the plane; that is, $f$ vanishes identically and $c=0$. In this case we have $-\left(g_{y}\right)^{2}=g^{4}-(1+d) g^{2}+d=$ $\left(g^{2}-d\right)\left(g^{2}-1\right)$. The situation is conjugate to that of the preceding cases, the geometric interpretation of horizontal and vertical paths being interchanged. The surfaces are well defined on a horizontal strip bounded by the set $D=\{(x, y) \in$ $\mathbb{R}^{2}: y=b_{0}$ and $\left.y=b_{1}\right\}$ (with $g^{2}\left(b_{0}\right)=g^{2}\left(b_{1}\right)=1$ ).

(3a) $d>1$. The set $D$ is two straight lines. $A$ is a horizontal strip but the image is an annulus foliated infinitely and having the third coordinate $y$ bounded in $\mathbb{H}^{2} \times \mathbb{R}$ (each level curve has curvature greater than one). The horizontal curves have constant curvature $k_{g}\left(\gamma_{h}\right)=g\left(y_{0}\right)>1$ and they are periodic in $x \in \mathbb{R}$. The curves $\gamma_{h}$ are circles with curvature greater than one. These surfaces are the rotationally invariant catenoids described in [Nelli and Rosenberg 2002]. They are bounded by two parallel horizontal circles at infinity in the cylinder model of $\mathbb{H}^{2} \times \mathbb{R}$.

(3b) $0<d \leq 1$. Here $A$ is a strip, but the horizontal curve has constant curvature less than one. The horizontal curves are not compact: they are equidistant curves. The third coordinate $y$ is bounded. When $g^{2}\left(y_{0}\right)=c$, we have $\omega\left(y_{0}\right)=0$ and the tangent plane of $A$ is vertical along this curve of curvature: $k_{g}=\sqrt{c}<1$. We can assume that $L=\mathbb{T}^{2} \times\{0\}$ is a plane of symmetry. The equidistant curves deform and disappear at infinity. But since $\sinh \omega=\left(1+g^{2}\right)^{-1} g_{y}$ has no change of sign above the plane of symmetry, the horizontal horocycles are contained in the nonconvex side of $(L \cap A) \times \mathbb{R}$. The surfaces converge to a geodesic vertical plane as $c \rightarrow 0$. 
(3c) $d<0$. Here $g \in[-1,1]$. The horizontal level curves are not compact. There is $y_{0}$ (which we can assume to be 0 ) such that $g\left(y_{0}\right)=0$. The corresponding level curve is a geodesic which we can assume passes through the center of the disc in the Poincaré model. But along this curve $\omega$ is never zero and the tangent plane to the surface is never vertical. The surface is a global graph on $\mathbb{T}^{2}$ with third component $y$ bounded, and it is foliated by equidistant curves.

(4) The annulus family. Here we assume $c<0$ and $d>0$. The behavior of $f^{2}$ and $g^{2}$ is described in Figure 2, top. The important fact is that $Y_{-}>0$, that is, $\sqrt{\Delta}<1+d-c$. The value of $g^{2}$ oscillates between $Y_{-}$and $Y_{+}$. The set $B$ is represented by the straight line $X+Y=1$ in the plane $(X, Y)$ of Figure 2, top. We notice the interesting property

$$
X_{-}+Y_{+}=1 \quad \text { and } \quad X_{+}+Y_{-}=1 .
$$

The function $f^{2}$ has values in $\left[0, X_{+}\right]$. If $d-c \geq 1$ then $2 X_{-}=1+c-d-\sqrt{\Delta}<0$. When $d-c \leq 1$ we have $\sqrt{\Delta}>1+c-d$, which is $X_{-}<0$. Then the set $B^{+}$ contains the horizontal strip $\left\{(x, y) \in \mathbb{R}^{2}: 1<g^{2}\right\}$. On this strip, the horizontal curves have curvature greater than one and they are infinite coverings of a circle. The strip covers the annulus with the period of the function $f$. The horizontal curve $g^{2}=1$ is a horocycle having one point at infinity, parametrized by one point of the set $D$. The set $B^{-}$is a countable set of disks (see Figure 2, top), each tangent to two others. Since $f$ is an oscillating function between $-\sqrt{X_{+}}$ and $\sqrt{X_{+}}$, the sign of $f_{x}$ is alternately positive and negative, and one can see that $f_{x}+g_{y}=0$ or $f_{x}-g_{y}=0$ on the half-boundary of each disk $B^{-}$. Then $D$ is a set of disconnected curves homeomorphic to $\mathbb{R}$, and disconnects $\mathbb{R}^{2}$ into connected components homeomorphic to strips (see Figure 2).

On each period, there exist $x_{0}$ and $x_{1}$ with $f\left(x_{0}\right)=f\left(x_{1}\right)=0$. The curve $X\left(x_{0}, y\right)$ has the same point at infinity as one of the horocycles $\left(g^{2}=1\right)$, and intersects the other horocycle orthogonally. The curve $X\left(x_{0}, y\right)$ projects to a geodesic having the same points at infinity as the horocycles. We have to determine whether the endpoints of $X\left(x_{0}, y\right)$ project to the same point or to the two endpoints of the geodesic. This depends of the sign of $\omega$, which determines the vertical component of the Gauss map. If $\omega$ has no change of sign, the curve $X\left(x_{0}, y\right)$ is a graph on the geodesic and then the projection of $X\left(x_{0}, y\right)$ has two points at infinity. If $\omega$ is positive and then negative, $X\left(x_{0}, y\right)$ projects to a half-geodesic and there is only point at infinity (as in a catenoid). When $X\left(x_{0}, y\right)$ has the same points at infinity as one horocycle $H_{1}$, the curves $X\left(x_{1}, y\right)$ and $H_{1}$ are orthogonal at their intersection. Then $X\left(x_{0}, y\right)$ and $X\left(x_{1}, y\right)$ project onto the same geodesic $\gamma$. The vertical plane $\gamma \times \mathbb{R}$ is a plane of symmetry. 
If $\omega=0$, then $f_{x}+g_{y}=0$. Recall that $f_{x}^{2}-g_{y}^{2}=\left(g^{2}+f^{2}-1\right)\left(g^{2}-f^{2}+c-d\right)$. Since $f$ vanishes on $X\left(x_{0}, y\right)$, we are looking for points where $g^{2}=d-c>0$. Since $g^{2}$ has values in $\left[Y_{-}, Y_{+}\right]$with $Y_{+}=\frac{1}{2}(1+d-c+\sqrt{\Delta})$, we have $Y_{+}-(d-c)=$ $\frac{1}{2}(1+c-d+\sqrt{\Delta})=X_{+}>0$ and $Y_{-}-(d-c)=\frac{1}{2}(1+c-d-\sqrt{\Delta})=X_{-}<0$. Assume $d-c \neq 1$. We have two zeros of $f_{x}^{2}-g_{y}^{2}$, which lie on $B^{-}$or $B^{+}$. Since $g$ is oscillating, $g_{y}$ changes sign at these two points while $f_{x}$ is constant. At one of these points $f_{x}+g_{y}$ vanishes and at the other $f_{x}-g_{y}$ vanishes. There is only one of these points where $\omega=0$. By analyzing the limit at infinity of $\sinh \omega=\left(f_{x}+g_{y}\right) /\left(f^{2}+g^{2}-1\right)$ at the neighborhood of $D$ we can see that $\omega$ changes sign. The analysis is similar in the case $d=c+1$. This leads us to expect the behavior of an annulus as in Figure 2, bottom, having two circles at infinity not homologous to zero in the cylinder's boundary of $\mathbb{H}^{2} \times \mathbb{R}$.

(5) The undulated helicoidal family. Here we assume $c>0$ and $d<0$. The behavior of $f^{2}$ and $g^{2}$ is depicted in Figure 3. The important fact is that $X_{-}>0$, that is, $\sqrt{\Delta}<1+c-d$. Then $f^{2}$ oscillates between $X_{-}$and $X_{+}$. The set $B$ is represented by the straight line $X+Y=1$ in the plane $(X, Y)$ of Figure 3 . We note the interesting property

$$
X_{-}+Y_{+}=1 \quad \text { and } \quad X_{+}+Y_{-}=1 .
$$

The function $g^{2}$ takes values in $\left[0, Y_{+}\right]$in this case. If $c-d \geq 1$ then $2 Y_{-}=$ $1+d-c-\sqrt{\Delta}<0$. When $c-d \leq 1$ we have $\sqrt{\Delta}>1+d-c$ which is $Y_{-}<0$. Then the set $B^{+}$contains the vertical strip $\left\{(x, y) \in \mathbb{R}^{2}: 1 \leq f^{2} \leq Y_{+}\right\}$. Each horizontal curve has curvature less than one (since $g^{2} \leq Y_{+}<1$ ). The surface is simply connected. The curves $\{g=0\}$ are geodesics having the same points at infinity. To see that, we note that the vertical curve $\{f=1\}$ projects to curves $\gamma_{v}$ ending at $p_{1}^{\prime}$ and $p_{2}^{\prime}$, points at infinity in $\mathbb{W}^{2}$ of two geodesics. The curve $\gamma_{v}$ has curvature $\left|k_{g}\left(\gamma_{v}\right)\right|=|\operatorname{coth} \omega|>1$ and crosses a geodesic orthogonally at a point $p_{0}$. By the maximum principle, $\gamma_{v}$ is contained in the convex part of a horocycle passing through $p_{0}$ and orthogonal to the geodesic at that point. Then $\gamma_{v}$, the horocycle and the geodesic passing through $p_{0}$ have the same point at infinity: $p_{0}^{\prime}=p_{1}^{\prime}=p_{2}^{\prime}$. The same holds for $p_{0}^{\prime \prime}=p_{1}^{\prime \prime}=p_{2}^{\prime \prime}$. This proves that the surface has a vertical period. The geodesics are lines of symmetry of the surface.

The horizontal curves between two geodesics have curvature less than one. The boundary at infinity of the surface has two connected components, each a copy of $\mathbb{R}$. They separate the cylinder $S^{1} \times \mathbb{R}$ into two connected components $A$ and $B$. Consider a Euclidean unit normal vector $n$ on such a curve, pointing into, say, component $A$. If $n$ points up at both $p_{1}^{\prime}$ and $p_{0}^{\prime}$ then the curve at infinity spirals around; that is, the projection of the curve on $S^{1} \times\{0\}$ is not homologous to zero. 

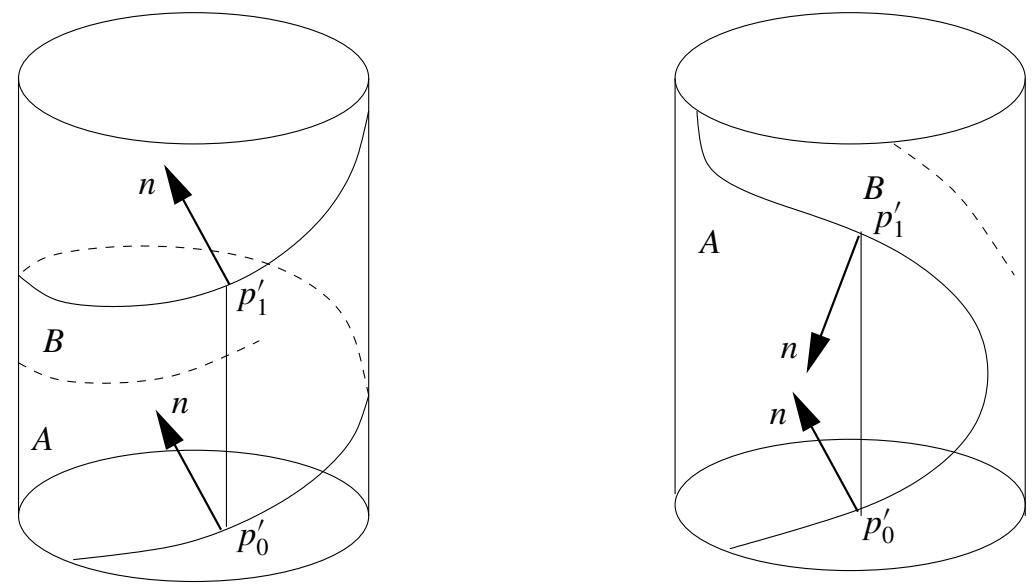

Figure 7. The undulated helicoid at infinity.

If $n$ points in opposite directions at $p_{0}^{\prime}$ and $p_{1}^{\prime}$, the projection is homologous to zero (Figure 7) and the surface is homologous to a vertical plane $\gamma \times \mathbb{R}$.

In $\mathbb{H}^{2}$, we have $\phi_{y}=(\sinh \omega / \sqrt{\rho}) e^{i \psi}$ and $\left|\phi_{y}\right|_{\rho}^{2}=|\sinh \omega|^{2} \rightarrow \infty$ when the curve is going to infinity. Then the tangent plane is becoming horizontal and the unit normal vector to the surface is pointing up or down. In fact, if $\left(N_{h}, N_{v}\right)$ is the unit normal vector with $N_{h}$ the horizontal component in $\mathbb{M}^{2}$ and $N_{v}=\tanh \omega$ the vertical component, the sign of $\omega$ will determine if $N$ is pointing down or up.

By construction (the surface is embedded), we have $\langle N, n\rangle \geq 0$ at infinity, then the sign of the limit in $D$ will tell us if we are spiraling or not on the cylinder.

One can see that $\omega=+\infty$ on one component and $-\infty$ in the other one, by analyzing the limit of

$$
\sinh \omega=\frac{f_{x}+g_{y}}{f^{2}+g^{2}-1}
$$

at the neighborhood of each component of $D$. The curves at infinity are spiraling and then we are describing an undulated helicoid.

(6) The case $c>0$ and $d>0$. We have

$$
\sqrt{\Delta}>|1+c-d| \text { and } \sqrt{\Delta}>|1+d-c|
$$

then $X_{-}>0$ and $Y_{-}>0$. The set $B^{+}$contains no strip in this case and we have two connected components homeomorphic to $\mathbb{R}$ in $D$ (see Figure 4). As in the preceding case we can see that $\omega=+\infty$ on one component and $\omega=-\infty$ on the other one. The curves are spiraling at infinity in a periodic way ( $\omega$ is periodic and so is the argument of $\phi_{x}$ ). The horizontal curves have curvature less than one at each level section but there is no horizontal geodesic in the surface. It is a blown-up helicoid. 
(7) The Riemann family. Here we assume $c<0$ and $d<0$. The behavior of $f^{2}$ and $g^{2}$ is depicted in Figure 5. We have $X_{-}<0$ (corresponding to $\sqrt{\Delta}>|1+c-d|$ ) and $Y_{-}<0(\sqrt{\Delta}>|1+d-c|)$. Then $f^{2} \in\left[0, X_{+}\right]$and $g^{2} \in\left[0, Y_{+}\right]$. The set $B$ is given by $X+Y=1$ and its inverse image disconnects the plane into one noncompact component and a countable set of disks. The set $B^{+}$contains the vertical strip $\left\{(x, y) \in \mathbb{R}^{2}: 1 \leq f^{2} \leq Y_{+}\right\}$and horizontal strip $\left\{(x, y) \in \mathbb{R}^{2}: 1 \leq g^{2} \leq X_{+}\right\}$. The sign of $f_{x}$ and $g_{y}$ gives the behavior of $D$ (see Figure 5) on the period. The horizontal strip $\left\{(x, y) \in \mathbb{R}^{2}: 1 \leq g^{2} \leq X_{+}\right\}$gives us an annulus bounded by two horocycles $k_{g}\left(\gamma_{h}\right)=g= \pm 1$ as in the annulus case.

Each vertical curve $\{f=0\}$ has many connected components with end points on $D$. These curves project on geodesics in the horizontal section. We will prove that these curves project on only one geodesic $\gamma$ i.e. they are contained in exactly one vertical flat plane $(\gamma \times \mathbb{R})$ of symmetry of the surface.

First we prove that each connected component of $\{f=0\}$ projects on the whole geodesic $\gamma$. A first indication is the sign of

$$
\sinh \omega=\frac{f_{x}+g_{y}}{f^{2}+g^{2}-1},
$$

which does not change at $p_{0}$ and $p_{0}^{\prime}$ (Figure 5). The normal vector points up (or down) at the two endpoints. Recall that

$$
f_{x}^{2}-g_{y}^{2}=\left(g^{2}+f^{2}-1\right)\left(g^{2}-f^{2}+c-d\right) .
$$

If $g^{2} \neq 1$ and $f=0$, we have $f_{x}+g_{y}=0$ if and only if $g^{2}=d-c$. If $g^{2}=1$ and $f=0$ we have

$$
\sinh \omega=\frac{f_{x}+g_{y}}{g^{2}-1}=\frac{g^{2}+c-d}{f_{x}-g_{y}}=0
$$

if and only if $g^{2}=d-c$. Then if $d-c<0, \omega$ has constant sign on the vertical connected component of $\{f=0\}$ which is a graph on the geodesic (Figure 6). If $c=d$, then $\omega=0$ at one point $q_{1}=\{f=0\} \cap\{g=0\}$ but $\omega$ has a constant sign on the curve. If $d-c>0, g^{2}=d-c<Y^{+}$at two points and the sign of the vertical component of the normal changes twice (Figure 6). However $\omega$ has the same sign in the neighborhood of its end points. It projects (noninjectively) on the whole geodesic $\gamma$.

\section{Acknowledgments}

We are grateful to Harold Rosenberg, Pascal Collin and Frédéric Hélein for valuable conversations on the subject, and to Y. Fang for pointing out some mistakes in the first version of this paper. 
It is a pleasure to thank Silvio Levy for his editorial contribution to the last version of the paper.

\section{References}

[Abresch 1987] U. Abresch, "Constant mean curvature tori in terms of elliptic functions", J. Reine Angew. Math. 374 (1987), 169-192. MR 88e:53006 Zbl 0597.53003

[Akutagawa and Nishikawa 1990] K. Akutagawa and S. Nishikawa, "The Gauss map and spacelike surfaces with prescribed mean curvature in Minkowski 3-space”, Tohoku Math. J. (2) 42:1 (1990), 67-82. MR 91e:53010 Zbl 0679.53002

[Daniel 2004] B. Daniel, "Isometric immersions into $\mathbb{S}^{n} \times \mathbb{R}$ and $\mathbb{H}^{n} \times \mathbb{R}$ and applications to minimal surfaces”, preprint, 2004. math.DG/0406426

[Fang 1994] Y. Fang, “On minimal annuli in a slab”, Comment. Math. Helv. 69:3 (1994), 417-430. MR 95f:53014 Zbl 0819.53006

[Fang and Wei 1998] Y. Fang and F. Wei, “On uniqueness of Riemann's examples”, Proc. Amer. Math. Soc. 126:5 (1998), 1531-1539. MR 98j:53010 Zbl 0898.53006

[Hauswirth and Pacard 2005] L. Hauswirth and F. Pacard, "Minimal surfaces of finite genus with two limits ends", preprint, 2005. math.DG/0511438

[Jackson 1945] S. B. Jackson, "The four-vertex theorem for surfaces of constant curvature", Amer. J. Math. 67 (1945), 563-582. MR 7,259h Zbl 0060.34910

[Lawson 1980] H. B. Lawson, Jr., Lectures on minimal submanifolds, vol. 1, Second ed., Mathematics Lecture Series 9, Publish or Perish, Wilmington, DE, 1980. MR 576752 (82d:53035b) Zbl 0434.53006

[Meeks 1990] W. H. Meeks, "The theory of triply periodic minimal surfaces", Indiana Univ. Math. J. 39:3 (1990), 877-936. MR 92e:53012 Zbl 0721.53057

[Meeks and Rosenberg 1989] W. H. Meeks and H. Rosenberg, "The global theory of doubly periodic minimal surfaces”, Invent. Math. 97:2 (1989), 351-379. MR 90m:53017 Zbl 0676.53068

[Meeks and Rosenberg 2004] W. H. Meeks and H. Rosenberg, "Stable minimal surfaces in $M \times \mathbb{R}^{\text {", }}$ J. Differential Geom. 68:3 (2004), 515-534. MR 2006b:53007 Zbl 0948.65107

[Meeks and Rosenberg 2005] W. H. Meeks and H. Rosenberg, "The theory of minimal surfaces in $M \times \mathbb{R} "$, Comment. Math. Helv. 80:4 (2005), 811-858. MR MR2182702 Zbl 0948.65107

[Meeks et al. 1998] W. H. Meeks, J. Pérez, and A. Ros, "Uniqueness of the Riemann minimal examples”, Invent. Math. 133:1 (1998), 107-132. MR 99b:53013 Zbl 0916.53004

[Meeks et al. 2004] W. H. Meeks, J. Pérez, and A. Ros, "The geometry of minimal surfaces of finite genus, I: Curvature estimates and quasiperiodicity”, J. Differential Geom. 66:1 (2004), 1-45. MR 2006a:53007 Zbl 1068.53012

[Nelli and Rosenberg 2002] B. Nelli and H. Rosenberg, "Minimal surfaces in $\mathbb{H}^{2} \times \mathbb{R}^{\prime}$, Bull. Braz. Math. Soc. (N.S.) 33:2 (2002), 263-292. MR 2004d:53014 Zbl 1038.53011

[Pedrosa and Ritoré 1999] R. H. L. Pedrosa and M. Ritoré, "Isoperimetric domains in the Riemannian product of a circle with a simply connected space form and applications to free boundary problems", Indiana Univ. Math. J. 48:4 (1999), 1357-1394. MR 2001k:53120 Zbl 0956.53049

[Rosenberg 2002] H. Rosenberg, "Minimal surfaces in $\mathbb{M}^{2} \times \mathbb{R}^{\prime}$, Illinois J. Math. 46:4 (2002), 11771195. MR 2004d:53015 Zbl 1036.53008

[Sá Earp and Toubiana 2004] R. Sá Earp and E. Toubiana, "Screw motion surfaces in $\mathbb{H}^{2} \times \mathbb{R}$ and $\mathbb{S}^{2} \times \mathbb{R}$ ", preprint, Pontífica Universidade Católica, Rio de Janeiro, 2004. 
[Schoen and Yau 1997] R. Schoen and S. T. Yau, Lectures on harmonic maps, Conf. Proc. and Lecture Notes in Geometry and Topology 2, International Press, Cambridge, MA, 1997. MR 98i:58072 Zbl 0886.53004

[Shiffman 1956] M. Shiffman, "On surfaces of stationary area bounded by two circles, or convex curves, in parallel planes", Ann. of Math. (2) 63 (1956), 77-90. MR 17,632d Zbl 0070.16803

[Wan 1992] T. Y.-H. Wan, "Constant mean curvature surface, harmonic maps, and universal Teichmüller space”, J. Differential Geom. 35:3 (1992), 643-657. MR 94a:58053 Zbl 0808.53056

Received July 2, 2004. Revised January 31, 2005.

LAURENT HAUSWIRTH

UNIVERSITÉ DE MARNE-LA-VALLÉE

5, CITÉ DESCARTES

CHAMPS-SUR-MARNE

77454 MARNE-LA-VALLÉE

FRANCE

hauswirth@univ-mlv.fr 